\title{
Hippocampal-medial prefrontal circuit supports memory updating during learning and post-encoding rest
}

\author{
Margaret L. Schlichting ${ }^{1}$ \& Alison R. Preston ${ }^{1-3^{*}}$ \\ ${ }^{1}$ Center for Learning and Memory and \\ Departments of ${ }^{2}$ Psychology and ${ }^{3}$ Neuroscience
}

The University of Texas at Austin,

1 University Station C7000

Austin, TX 78712

Phone: (512) 232-5145

Fax: (512) 475-8000

Running title: Memory updating during learning and rest

${ }^{*}$ Corresponding author:
Alison R. Preston, Ph.D.
Center for Learning \& Memory
The University of Texas at Austin
1 University Station, C7000
Austin, TX 78712
apreston@utexas.edu 


\section{ABSTRACT}

Learning occurs in the context of existing memories. Encountering new information that relates to prior knowledge may trigger integration, whereby established memories are updated to incorporate new content. Here, we provide a critical test of recent theories suggesting hippocampal (HPC) and medial prefrontal (MPFC) involvement in integration, both during and immediately following encoding. Human participants with established memories for a set of initial (AB) associations underwent $f M R I$ scanning during passive rest and encoding of new related $(\mathrm{BC})$ and unrelated $(\mathrm{XY})$ pairs. We show that HPCMPFC functional coupling during learning was more predictive of trial-by-trial memory for associations related to prior knowledge relative to unrelated associations. Moreover, the degree to which HPC-MPFC functional coupling was enhanced following overlapping encoding was related to memory integration behavior across participants. We observed a dissociation between anterior and posterior MPFC, with integration signatures during post-encoding rest specifically in the posterior subregion. These results highlight the persistence of integration signatures into post-encoding periods, indicating continued processing of interrelated memories during rest. We also interrogated the coherence of white matter tracts to assess the hypothesis that integration behavior would be related to the integrity of the underlying anatomical pathways. Consistent with our predictions, more coherent HPC-MPFC white matter structure was associated with better performance across participants. This HPC-MPFC circuit also interacted with content-sensitive visual cortex during learning and rest, consistent with reinstatement of prior knowledge to enable updating. These results 
show that the HPC-MPFC circuit supports on- and offline integration of new content into memory.

\section{KEYWORDS}

hippocampus; medial prefrontal cortex; memory integration; inference; connectivity; diffusion-weighted imaging 


\section{INTRODUCTION}

Episodic memories do not exist in isolation, but rather influence one another in important ways (Moscovitch et al., 2005; Nadel et al., 2012). For example, new learning events that relate to prior knowledge may trigger reactivation of the existing memory, which may then be modified as a function of the current experience (Nader et al., 2000b; Hupbach et al., 2007; Nader and Einarsson, 2010; Kuhl et al., 2012; Zeithamova et al., 2012a; Gershman et al., 2013; Schlichting and Preston, 2014). In addition to allowing for outcomes like memory distortion (Loftus, 2005; Hupbach et al., 2007; Gershman et al., 2013) and deletion (Nader et al., 2000a), such retrieval may also benefit new learning. By recalling related memories during a new experience, prior knowledge may serve as a foundation that facilities encoding of the new content (Bartlett, 1932), thereby allowing memories to be linked across time. Such integration is thought to underlie the formation of complex knowledge structures like memory networks (Eichenbaum et al., 1999) or schema (van Kesteren et al., 2012; Ghosh and Gilboa, 2013; Preston and Eichenbaum, 2013).

Recent theory has implicated hippocampal (HPC)-medial prefrontal (MPFC) interactions in this process, with MPFC guiding HPC encoding and retrieval when new information can be incorporated into existing knowledge (van Kesteren et al., 2012; Preston and Eichenbaum, 2013; Schlichting and Preston, 2015). We hypothesize that this circuit may be similarly engaged during offline periods to promote integration across episodes (Lewis and Durrant, 2011), with integrated memories ultimately stored in MPFC (Schlichting et al., 2015). While empirical research has broadly demonstrated involvement of HPC and MPFC in memory integration (Tse et al., 2007, 2011; van 
Kesteren et al., 2010a; Zeithamova et al., 2012a), existing reports diverge in their main findings. While some have shown HPC-MPFC connectivity increases during events associated with strong prior knowledge (Tse et al., 2011; Zeithamova et al., 2012a), others have observed decreases (van Kesteren et al., 2010a), leaving open questions as to how these findings may fit together under a single mechanism. More evidence is needed to inform our understanding of when and how the HPC-MPFC circuit supports encoding.

Here, we seek to fill this gap in understanding by providing a controlled test of theories regarding interregional interactions during integration (van Kesteren et al., 2012; Preston and Eichenbaum, 2013), particularly focusing on HPC and MPFC as our a priori regions of interest. We propose that integration through HPC-MPFC communication reduces interference among related memories, facilitating encoding of overlapping experiences. We manipulate the overlap between prior knowledge and new learning on a memory-by-memory basis, allowing for the examination of HPC-MPFC interactions mediating updating of individual events. Moreover, the hetereogeneity of MPFC has been underappreciated in prior research on this topic; differences in structural connectivity across MPFC (Ongür and Price, 2000) suggest that its posterior aspects might be particularly important for integration (Nieuwenhuis and Takashima, 2011; Schlichting et al., 2015). We formally test this idea by interrogating neural signatures within subregions of MPFC.

Here, we provide a targeted investigation of HPC and MPFC contributions to integration both across and within individuals, comparing on- and offline neural engagement during periods reflecting memory updating versus simple associative 
encoding. Behavioral studies in humans have revealed that passive rest following learning promotes memory for individual episodes (Melton, 1970; Cowan, 2004; Dewar et al., 2012; Craig et al., 2015a), perhaps in allowing for the engagement of early consolidation processes (McClelland et al., 1995). Recent work has extended these findings to demonstrate sleep- (Ellenbogen et al., 2007; Coutanche et al., 2013) and rest- (Craig et al., 2015b) related increases in performance on novel judgments spanning multiple experiences, consistent with the idea that offline processing may also facilitate integration (Buckner, 2010). Mechanistically, sleep-based memory reactivation triggered by HPC sharp wave ripples has been hypothesized to enable connections to be formed among co-activated representations, promoting integration (Lewis and Durrant, 2011) in functionally coupled neocortical regions like MPFC (Wierzynski et al., 2009). Inspired by such theories, we propose that similar reorganizational processes may occur during awake rest periods immediately following learning via HPC-MPFC interactions. While sharp wave ripples are known to occur predominantly during slowwave sleep (Diekelmann and Born, 2010), empirical work in rodents and humans suggests that HPC ripples also occur during awake rest following learning (Buzsaki et al., 1983; Axmacher et al., 2008; Ego-Stengel and Wilson, 2010; Cornwell et al., 2014). While the behavioral significance of such awake-phase events remains unclear (see however Axmacher et al., 2008), it may be the case that like ripples events during sleep, rest-phase ripples also trigger reactivation of prior experience (Foster and Wilson, 2006; Karlsson and Frank, 2009; Gupta et al., 2010; Staresina et al., 2013; Schlichting and Preston, 2014) and allow for integration across multiple related memories (Craig et al., 2015b) activated simultaneously in the brain. We thus predicted that HPC-MPFC 
interactions would be enhanced during rest periods following opportunities for memory updating, with the degree of enhancement predicting integration-related behaviors. Furthermore, as behaviors tapping the HPC-MPFC circuit should depend on the integrity of the underlying structural connections, we hypothesized that more coherent white matter tracts connecting these regions would be associated with superior integration.

\section{MATERIALS AND METHODS}

Data from some portions of this experiment were described in a previous report (Schlichting and Preston, 2014) examining how processes prior to encoding influence new learning. Here, we focus on how the HPC-MPFC circuit is engaged during and immediately following new learning experiences.

\subsection{Participants}

Participants were as reported previously (Schlichting and Preston, 2014). Fortyeight right-handed volunteers (27 females; ages $20-33$, mean \pm SEM $=24.6 \pm 0.5$ years) participated in the experiment. Consent was obtained in accordance with an experimental protocol approved by the Institutional Review Board at the University of Texas at Austin. Participants received monetary compensation for their involvement in the study. Data from a total of 13 participants were excluded for the following reasons: hardware malfunction $(N=5)$, handedness concerns $(N=1)$, and low memory performance $(N=7)$. Low memory performance was defined as either (a) failure to subsequently recall more than $10 \%$ of $\mathrm{BC}$ and $\mathrm{XY}$ associations studied in the scanner 
$(\mathrm{N}=6)$ or (b) failure to reach near-perfect performance on initial $A B$ associations (< $80 \%$ cued recall accuracy; $N=1$ ). Data from the remaining 35 participants were included in all functional analyses (21 females; ages 20-30, $24.1 \pm 0.5$ years). An additional 10 participants were excluded from the diffusion tensor imaging (DTI) analysis due to data acquisition error. Thus, 25 participants were included in the structural connectivity analysis (15 females; ages $20-30,24.2 \pm 0.7$ years).
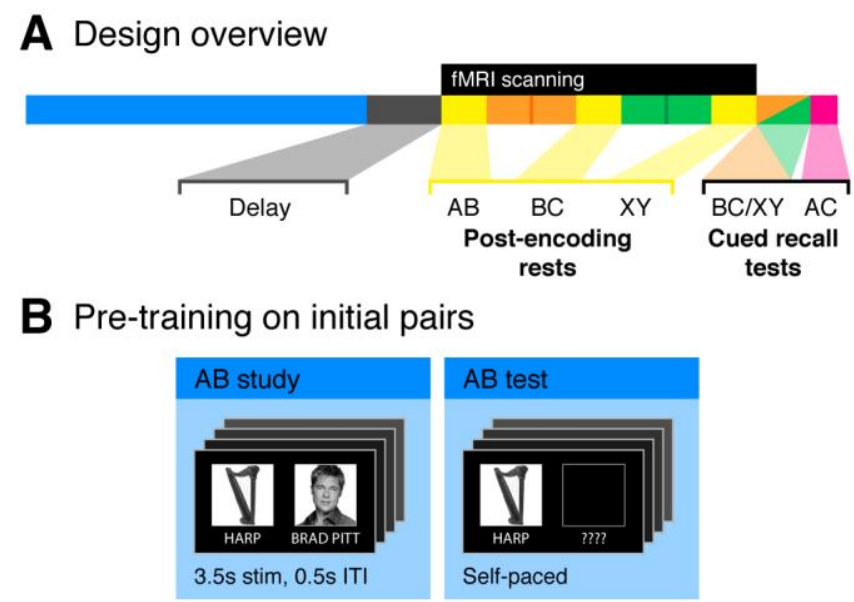

C New pair learning (scanned)
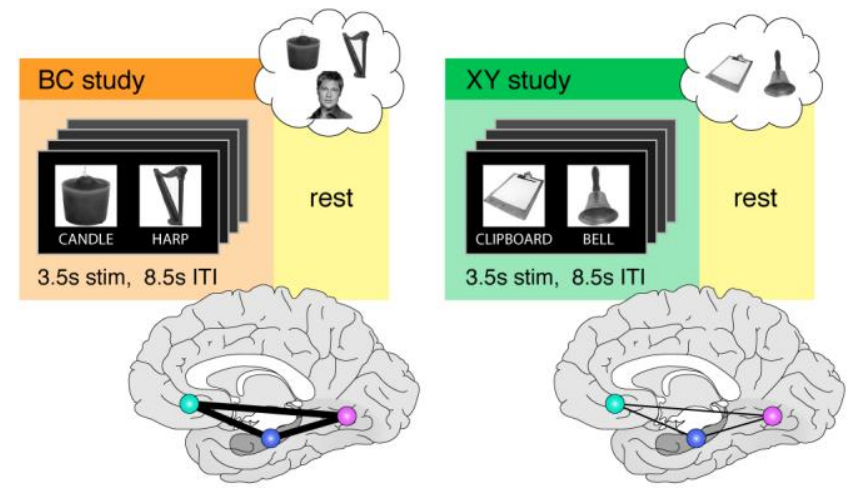

Figure 1. Experimental design and theoretical predictions. (A) Schematic depiction of the experiment timeline. The present paper focuses on the BC (orange) and XY (green) encoding phases and post-encoding rest periods (yellow). See also panel C. For findings related to the post-AB rest period, see Schlichting \& Preston 2014. (B) Participants learned a series of $A B$ face-object associations across four alternating study-test repetitions during the pre-training phase. Memory performance was near ceiling by the final test block, demonstrating strong memories for the $A B$ pairs (see 
Results, Behavioral performance). (C) Predictions for the present study. Following the pre-training phase, participants were transferred to the fMRI scanner for study of overlapping $\mathrm{BC}$ and non-overlapping $\mathrm{XY}$ object-object associations. $\mathrm{BC}$ and $\mathrm{XY}$ study blocks were each followed by a rest period (yellow). The order of $B C$ and $X Y$ study was counterbalanced across participants. Left, overlapping BC associations (orange) included one object (here, HARP) that had previously been paired with a face during the pre-training phase (panel $A$, blue). Such overlap allows for prior $A B$ memories to be reactivated and updated with the new $\mathrm{BC}$ information. We hypothesize that this process engages a network comprising hippocampus (HPC, indigo), medial prefrontal cortex (MPFC, teal), and content-sensitive visual regions (e.g., fusiform face area [FFA], magenta). Right, non-overlapping $X Y$ associations (green) included two new objects that had not been previously learned during the pre-training phase. Brain schematics represent the predicted functional connectivity enhancement among HPC, MPFC and FFA for overlapping BC (thick black lines) relative to XY (thin black lines) study and post-encoding rest periods.

\subsection{Materials}

Stimuli for the associative inference task (Fig. 1) (Preston et al., 2004; Zeithamova and Preston, 2010; Zeithamova et al., 2012a) consisted of 60 grayscale images of famous faces ( 30 male and 30 female) and 240 grayscale images of common objects organized into 60 triads (denoted $\mathrm{ABC}$ ) and 60 pairs (denoted $\mathrm{XY}$ ). All images were presented with verbal labels. $A B C$ triads consisted of one face and two objects and were presented as overlapping $A B$ and $B C$ pairs, with the $B$ item shared between pairs. That is, $A B$ pairs always consisted of one famous face $(A)$ paired with one object $(B)$

(Fig. 1B); the same object (B) was then paired with a different object $(C)$ to form a BC pair (Fig. 1C, left). Non-overlapping XY pairs consisted of two objects (Fig. 1C, right). All items were unique to their triad or pair, such that a single face or object image was a member of only one $A B C$ triad or one $X Y$ pair. Four randomization groups were created to control for the organization of images into triads and pairs and viewing order. Objects were randomly assigned to item type $(\mathrm{B}, \mathrm{C}, \mathrm{X}$ or $\mathrm{Y})$, which determined both whether it belonged to a triad or pair and during which phase(s) it served as a recall cue (see 
below). An equal number of $B C$ pairs associated with male and female faces $(A)$ were presented within each of two BC encoding scans; no other constraints in item assignment or trial order were imposed. As described below, the order of $\mathrm{BC}$ encoding versus $\mathrm{XY}$ encoding was counterbalanced across participants.

\subsection{Memory task}

An overview of the experimental design is provided in Fig. 1A. Prior to scanning, participants were trained to near-perfect performance on all $60 \mathrm{AB}$ (face-object) pairs (Fig. 1B). The goal of the pre-training phase was to create established memories for the $A B$ pairs, such that overlapping $B C$ information could then be encoded in relation to strong existing memories. The $A B$ pre-training phase consisted of 4 study-test alternations. During the study phase, participants viewed each AB pair once (Fig. 1B, left; $3.5 \mathrm{~s}$ stimulus, $0.5 \mathrm{~s}$ ITI). A items (faces) were always shown on the right; $B$ items (objects) were always on the left. Participants were encouraged to construct a visual or verbal story linking the items to aid memory but were not required to make any explicit response. Each study phase in the pre-training portion of the experiment lasted 4 minutes.

Following each study phase, participants completed a self-paced cued recall test (Fig. 1B, right). The B item (object) was presented on the left side of the screen next to an empty box. Participants were asked to say aloud the name of the face that was paired with it. After either a verbal response had been produced or the trial was "passed," participants viewed a feedback display in which the correct image appeared in

place of the empty box. Including the feedback displays, each pair was viewed a total of 
8 times during the pre-training phase.

After completing the initial $A B$ pair pre-training, participants were transferred to the scanner. At no time were participants made aware by the experimenter of the relationship between the pre-training phase and subsequent study and test tasks (Schlichting and Preston, 2014). Once in the scanner, fMRI data was collected during 6 minutes of passive rest. During this time, a white fixation cross was displayed on a black screen. Participants were instructed to think about whatever they liked while remaining awake and alert with their eyes open.

Following the initial post-AB rest scan, participants were scanned during encoding of overlapping BC (Fig. 1C, left) and non-overlapping XY pairs (Fig. 1C, right). Pairs were segregated by type into separate scans. There were a total of four slow eventrelated scans (2 BC scans and $2 \mathrm{XY}$ scans; 3.5 seconds stimulus, 8.5 seconds ITI). Participants were encouraged to construct a visual or verbal story while they encoded the new associations; no explicit responses were required. Each pair was presented just once, requiring rapid acquisition of associative information. $C$ and $Y$ objects were on the left; $\mathrm{B}$ and $\mathrm{X}$ objects were on the right. BC study scans always occurred in immediate succession, as did $X Y$ study scans. Encoding order of $B C$ and $X Y$ scans was counterbalanced across participants. That is, for half of the participants, all BC pairs (scans 1 and 2) were learned before XY pairs (scans 3 and 4); for the other half, the order was reversed. Each study scan was 6 minutes long.

Post-encoding rest scans were acquired immediately following BC (e.g., after study scan 2) and XY (e.g., after study scan 4) learning (Fig. 1C, yellow). These scans were identical to the post-AB encoding rest scan described previously. Participants 
were instructed to think about whatever they liked during the post-encoding rest scans. Importantly, though participants were aware of the overlap between $A B$ and $B C$ associations, they had no reason to believe they would be tested on AC inference associations (in fact, only two participants reported anticipating the AC inference test; see below). Therefore, while explicit rehearsal of the $\mathrm{BC}$ or $\mathrm{XY}$ pairs is a possibility during these rest scans, it is unlikely that participants would intentionally rehearse the prior related $\mathrm{AB}$ knowledge.

After the final rest scan, participants completed a cued recall test on $B C$ and $X Y$ pairs (Fig. 1A). This occurred outside of the scanner; no imaging data was collected during the test. $C$ and $Y$ items, presented on the left, served as probes. $B C$ and $X Y$ test trials were randomly intermixed. Following completion of the $\mathrm{BC} / \mathrm{XY}$ test, structure of the inferential (AC) associations was explained to participants. That is, participants were told that $A$ and $C$ items both paired with the same $B$ item were indirectly related. Only two participants reported that they anticipated this inference test, even though all participants became aware of the overlap between the $A B$ and $B C$ associations during the BC study phase. They then completed a cued recall test on these surprise inference associations. The same items $(C)$ served as probes, but this time participants were asked to name the indirectly related item (A, always a face). Importantly, BC and XY associations were of the same content type; they differed only in the degree to which they could be incorporated into prior $(\mathrm{AB})$ memories. For this reason, we focus primarily on the comparison between $\mathrm{BC}$ and $\mathrm{XY}$ memory as an index of memory integration; however, we also provide additional follow-up analyses detailing the relationship between neural signatures and subsequent AC performance (notably, AC and BC 
performance were highly correlated). No feedback was provided during post-scanning $\mathrm{BC} / \mathrm{XY}$ or $\mathrm{AC}$ inference tests to prevent additional learning of the direct $(\mathrm{BC} / \mathrm{XY})$ associations.

Participants had the opportunity to practice the memory task before beginning the experiment. The practice included only non-overlapping face-object associations using different stimuli from the main experiment.

\subsection{Analysis of behavioral data}

Cued recall responses were hand scored. Responses were scored as correct if the participant produced the correct label or, for famous faces, if they provided a unique and accurate description of the person (e.g., by naming a film in which the actor was featured). We used this liberal criterion for recall because we found that participants would often recall a stimulus in great detail despite an inability to remember the specific verbal label. This type of recall performance was true for virtually only the $A_{\text {face }}$ stimuli, and was particularly common early in the initial $A B$ pair pre-training. For example, instead of recalling Daniel Radcliffe, the participant may state "the guy who plays Harry Potter." This criterion has been employed in prior studies using a similar stimulus set and paradigm (Kuhl et al., 2011). A proportion correct was calculated for each participant, pair type (for $A B, B C, X Y$ and $A C$ ) and repetition (for $A B$ only).

As reported previously (Schlichting and Preston, 2014), performance on BC pairs and $A C$ inferences was highly correlated, both across participants $\left(r_{33}=0.98\right)$ and on a triad-by-triad basis within participant. $A B$ performance also predicted both memory for 
the overlapping $B C$ information and $A C$ inference performance. For additional behavioral analyses, see Schlichting and Preston, 2014.

\subsection{Visual localizer task}

After the memory task, participants completed a blocked design functional localizer during $f M R I$ scanning to obtain neural patterns associated with viewing different types of visual stimuli. Participants viewed blocks of faces, objects, and scrambled objects while performing a 1-back task. For each image, they pressed one of two buttons on a keypad to indicate whether the picture was new or a repeat of the immediately preceding picture. Responses were collected solely to ensure attention to the task and were not considered as part of the analysis. The images used in the localizer task were different from those used in the memory task. There were four blocks of each stimulus type per run, plus additional interleaved blocks of passive fixation. Blocks were 18 seconds long, yielding a total run length of 5 minutes. Three localizer scans were collected.

Participants had the opportunity to practice the visual localizer task before beginning the experiment. The practice contained one abbreviated block of each of the three stimulus types. Practice stimuli comprised images different from those used in the scanned task.

\subsection{MR data acquisition}

Imaging data were acquired on a 3.0T GE Signa MRI system (GE Medical Systems). All functional data were collected in 33, 3-mm thick oblique axial slices using an $\mathrm{EPI}$ sequence $(\mathrm{TR}=2000 \mathrm{~ms}, \mathrm{TE}=30.5 \mathrm{~ms}$, flip angle $=73 ; 64 \times 64$ matrix, $3.75 \mathrm{x}$ 
$3.75 \mathrm{~mm}$ in-plane resolution, bottom-up interleaved acquisition, $0.6 \mathrm{~mm}$ gap). T2weighted structural images were acquired in the same prescription as the functional images for the memory $(\mathrm{TR}=3200 \mathrm{~ms}, \mathrm{TE}=68 \mathrm{~ms}, 512 \times 512$ matrix, $0.46 \times 0.46 \mathrm{~mm}$ in-plane resolution) and visual localizer (TR $=3200 \mathrm{~ms}$, TE = $68 \mathrm{~ms}, 256$ × 256 matrix, $0.94 \times 0.94 \mathrm{~mm}$ in-plane resolution) tasks. Diffusion-weighted data were acquired to characterize white matter structure $(T R=12000 \mathrm{~ms}$, $\mathrm{TE}=87.1 \mathrm{~ms}, 25$ diffusion directions, maximum b-value $=1000,128 \times 128$ matrix, $0.94 \times 0.94$ in-plane resolution, 41 straight axial slices, 3-mm thickness, no gap). A T1-weighted 3D SPGR structural volume ( $256 \times 256 \times 172$ matrix, $1 \times 1 \times 1.3 \mathrm{~mm}$ voxels) was also collected to facilitate image coregistration and for automated parcellation using Freesurfer (http://surfer.nmr.mgh.harvard.edu/) (Desikan et al., 2006).

\section{7 fMRI data preprocessing}

Functional data were preprocessed using FSL version 5.0.2 (FMRIB's Software Library, http://www.fmrib.ox.ac.uk/fsl). The first 4 volumes of all functional scans were discarded to allow for T1 stabilization. Motion correction was performed within each functional scan using MCFLIRT by aligning all images in the run to the middle volume in the timeseries. Coregistration of functional data across runs was performed by calculating and applying the affine transformation from each run to a reference run using FLIRT, part of FSL. The 3D SPGR structural volume was registered to the functional reference run using the EPI registration utility (part of FLIRT) and then resampled to functional space. Brain extraction was performed on all structural and functional images using BET. 


\subsection{Regions of interest}

\subsubsection{Anatomical region of interest definition}

The HPC was delineated by hand on the $1 \mathrm{~mm} \mathrm{MNI}$ template brain and reversenormalized to each individual's functional space using Advanced Normalization Tools (ANTS) (Avants et al., 2011). Specifically, a non-linear transformation was calculated from the MNI template brain to each participant's 3D SPGR volume. This warp was then concatenated with the SPGR to functional space transformation calculated using FLIRT. After applying the transformation using ANTS, the anatomical HPC ROI was aligned to each participant's functional data.

MPFC ROls were generated for each participant using output from Freesurfer (Desikan et al., 2006) run on each individual's SPGR. Because previous studies on similar topics (Kumaran et al., 2009; van Kesteren et al., 2010b, 2014; Sweegers et al., 2013) have identified a range of activation foci throughout the medial surface of PFC, we combined medial orbitofrontal and rostroanterior cingulate to create an MPFC ROI for each participant. ROls were then aligned to each individual's functional data using transformations applied in FLIRT, as described above.

\subsubsection{Functional region of interest definition}

Functional data from the localizer task were used to define face-sensitive voxels within the fusiform gyrus (i.e., fusiform face area, FFA). Analysis of fMRI data from the localizer task was carried out using FEAT (fMRI Expert Analysis Tool) version 6.00, part of FSL. The following pre-statistics processing was applied: grand-mean intensity 
normalization of the entire $4 \mathrm{D}$ dataset by a single multiplicative factor; high-pass temporal filtering (Gaussian-weighted least-squares straight line fitting, with sigma $=64$ s); and spatial smoothing (5 mm FWHM). FILM prewhitening was used. Stimulus presentation blocks were modeled as events of $18 \mathrm{~s}$ duration, with one regressor for each stimulus type (face, object, scrambled object, passive fixation). Temporal derivates were included. Stimulus regressors were convolved with the canonical (double-gamma) HRF. Motion parameters calculated during the motion correction step and their temporal derivatives were added as additional confound regressors. Two measures of framewise data quality were also calculated to identify volumes that may be adversely impacted by motion artifacts: framewise displacement (FD) and DVARS (Power et al., 2012). FD measures the overall change in head position from one time point to the next, and is calculated by summing the absolute values of the derivatives of the six motion parameters calculated during the realignment step. DVARS measures the overall change in image intensity from one time point to the next. This index is calculated as the root mean square of the derivatives of the timecourses across all voxels in the brain. Both FD and DVARS were added to the model as regressors of no interest (Schonberg et al., 2014). Additional regressors were created for each time point in which motion exceeded a threshold of both $0.5 \mathrm{~mm}$ for FD and $0.5 \%$ change in BOLD signal for DVARS (plus one time point before and two time points after) (Power et al., 2012). Temporal filtering was then applied to the model.

After modeling functional data within each run, the resulting statistical images were combined across localizer runs for each participant using fixed effects. As data were already coregistered across runs, no additional registration or spatial normalization 
was necessary. Face-selective regions were defined for each participant as those voxels responding more to faces than objects and scrambled objects. The procedure for FFA definition was as follows: we created $14 \mathrm{~mm}$ spheres centered at each participant's peak voxel (i.e., the maximum z-statistic from the face $>$ objects + scrambled objects contrast image) located within the posterior half of their Freesurfer-defined fusiform gyrus. This sphere was then masked with fusiform gyrus to restrict FFA to gray matter voxels. This method was used to ensure FFAs of approximately the same size across participants. This procedure was carried out separately for the left and right hemispheres; lateralized ROls were then summed to create a bilateral FFA (ROI size range: $205-336$ functional voxels, mean $\pm \mathrm{SEM}=288.7 \pm 5.7$ voxels). As $\mathrm{ROI}$ definition took place in the native functional space of each participant, no realignment or resampling was necessary.

\subsection{Univariate analysis}

Analysis of fMRI data from the memory task was carried out using FEAT (fMRI Expert Analysis Tool) version 6.00, part of FSL. Grand-mean intensity normalization, high-pass temporal, filtering, spatial smoothing, and pre-whitening were carried out as described above.

We interrogated the relationship between encoding activation and subsequent memory using a general linear model (GLM). The GLM was designed to isolate effects specific to the encoding of new associations that overlapped with prior knowledge. Accordingly, encoding trials were sorted based on both subsequent memory and prior knowledge condition to create four conditions: BC encoding trials that were later correct, 
$\mathrm{BC}$ encoding trials that were later incorrect, $\mathrm{XY}$ encoding trials that were later correct, and $X Y$ encoding trials that were later incorrect. Contrasts of interest were (1) BC correct $>\mathrm{XY}$ correct and (2) BC correct $-\mathrm{BC}$ incorrect $>\mathrm{XY}$ correct $-\mathrm{XY}$ incorrect (i.e., the subsequent recall $\times$ prior knowledge interaction).

Stimulus presentations were modeled as events with $3.5 \mathrm{~s}$ durations, with one regressor for each condition of interest. The model was convolved with the canonical (double-gamma) HRF. Temporal derivatives were included. Motion parameters calculated during the motion correction step and their temporal derivatives were added as additional regressors of no interest. As described above, FD, DVARS, and individual regressors for time points exceeding FD and DVARS thresholds were added to the models to additionally account for motion effects (Power et al., 2012; Schonberg et al., 2014). Temporal filtering was applied to the model.

After modeling functional data within each run, the resulting statistics images were warped to the $\mathrm{MNI}$ template brain resampled to functional resolution $(3.75 \times 3.75 \times 3.6$ mm) using ANTS (Avants et al., 2011). The warped images were combined across encoding runs for each participant using fixed effects, and then across the group using mixed effects. For both contrasts of interest, correction for multiple comparisons was carried out at both the whole-brain level and using small volume correction within our a priori HPC and MPFC ROls. Correction for multiple comparisons at the whole brain level was carried out on group-level voxel-wise statistical images according to clusterbased Gaussian random field theory (Worsley et al., 2002), with a cluster-forming threshold of $z>2.3$ and a whole-brain corrected cluster significance level of $p<0.05$. We also interrogated activation within a priori HPC and MPFC ROls for integration- 
specific effects using small volume correction implemented in 3dClustSim, part of AFNI (Cox, 1996). Group statistics images were first masked with each ROI. We then applied a primary voxelwise threshold of $p<0.05$ to the masked images to identify those voxels within our ROls surpassing this initial p-value threshold. We then corrected for multiple comparisons within the ROls by determining the cluster extent corresponding to a cluster-corrected threshold of $p<0.05$ using Monte Carlo simulations. Cluster sizes that occurred with a probability of less than 0.05 across 2000 simulations were considered statistically significant (13 and 19 voxels at functional resolution for HPC and MPFC, respectively).

\subsection{Task-based functional connectivity}

Functional connectivity during task was examined using a psychologicalphysiological interactions (PPI; Friston et al., 1997; O'Reilly et al., 2012) approach carried out in FEAT version 6.00. Grand-mean intensity normalization, high-pass temporal, filtering, spatial smoothing, and pre-whitening were carried out as described previously.

The PPI analyses were performed to isolate regions whose functional connectivity with HPC and MPFC respectively were significantly modulated by the interaction of subsequent recall by prior knowledge condition. Encoding trials were sorted according to subsequent memory, and a correct - incorrect regressor was constructed for each run. Notably, because $B C$ and $X Y$ encoding trials occurred in different scans, this regressor represented the correct - incorrect difference for either BC or XY study trials, depending on the run. This served as the psychological regressor. The first eigenvariate 
of the filtered timeseries (derived from the univariate analyses described above) was extracted from each individual's anatomically defined HPC and MPFC and functionally defined FFA ROls and entered as the physiological regressors for the three PPI analyses. The PPI regressor served as the regressor of interest and was generated as the interaction between the psychological and physiological regressors. An additional task regressor was added to account for variance associated with both correct and incorrect trials.

For all three PPI models, stimulus presentations were modeled as events with 3.5 s durations. Task-related regressors and their temporal derivatives were convolved with the canonical (double-gamma) HRF and filtered. Physiological and PPI regressors were not convolved with the HRF or filtered, as these regressors were derived from neural signal that had previously undergone temporal filtering. Motion parameters calculated during the motion correction step and their temporal derivatives were added as additional regressors of no interest. As described above, FD, DVARS, and individual regressors for time points exceeding FD and DVARS thresholds were added to the models to additionally account for motion effects (Power et al., 2012; Schonberg et al., 2014).

After modeling functional data within each run, the statistics images associated with the PPI regressor were warped to the $\mathrm{MNI}$ template brain resampled to functional resolution using ANTS (Avants et al., 2011). The warped PPI images were contrasted for $B C$ and $X Y$ study runs for each participant using fixed effects, yielding a subsequent recall $\times$ prior knowledge interaction contrast (i.e., BC correct $-\mathrm{BC}$ incorrect $>\mathrm{XY}$ correct - $X Y$ incorrect). These statistics images were then combined across the group using 
mixed effects. Correction for multiple comparisons was performed within a priori HPC and MPFC ROls using small volume correction implemented in 3dClustSim as described above.

As our anatomical MPFC ROI spans a large and likely functionally diverse region (Ongür and Price, 2000; Price and Drevets, 2009; Roy et al., 2012), we also performed the PPI analysis described above using the MPFC clusters defined in the univariate interaction contrast (depicted in Fig. 2B) as the seed. This analysis was otherwise identical to above.

\subsection{Rest-phase functional connectivity}

Functional connectivity during rest was interrogated using a voxelwise regression approach with anatomically defined HPC and MPFC and functionally defined FFA as seed regions. This analysis approach is similar to that we have employed previously (Schlichting and Preston, 2014). We interrogated neural activation for clusters of voxels

(1) that showed enhanced rest-phase connectivity following overlapping (post-BC) relative to non-overlapping (post- $\mathrm{XY}$ ) encoding conditions and (2) for which the degree of enhancement was related to subsequent performance. Importantly, we would expect the post-XY rest period to reflect neural signatures related to simple associative encoding (i.e., of object-object pairs), Thus, using this period as a baseline provides a stringent control for isolating those processes related to memory integration above and beyond those engaged during learning more generally.

We first regressed out potential sources of noise from the resting state data. Specifically, we extracted the first eigenvariate of the signal across the duration of the 
each rest scan in anatomically defined white matter and lateral ventricle ROls. The signal from these two ROls and their temporal derivatives were used to construct a GLM along with: motion parameters, FD, DVARS, and their temporal derivatives; and timepoints with excessive motion (as described above). Rest data were high-pass filtered with a cutoff of $0.009 \mathrm{~Hz}$ (Fox et al., 2005; Tambini et al., 2010) and regressed on these noise sources. The resulting residual timeseries data for each participant were spatially smoothed (5 mm FWHM). As motion-related nuisance signals had already been removed, each model included only the seed $\mathrm{ROI}$ timeseries and its temporal derivative. The resulting parameter estimate image for each participant reflected the degree to which activation in each voxel tracked with activation in the seed ROI across each rest scan. We then warped these images to the $\mathrm{MNI}$ template resampled to functional dimensions using ANTS (Avants et al., 2011). Images were contrasted within participant (i.e., each participant's post-XY connectivity statistics image was subtracted from their post-BC connectivity image) and combined them across participants using a group-level GLM as follows.

We constructed the group-level model with both $\mathrm{BC}$ and $\mathrm{XY}$ performance as covariates. To identify those voxels showing enhanced connectivity with the seed region during post-BC relative to post-XY encoding (irrespective of memory performance), we combined the differences images across participants using a one-sample t-test. We also isolated voxels for which the degree of connectivity enhancements tracked more with $\mathrm{BC}$ than $\mathrm{XY}$ performance (i.e., contrast of covariates, $\mathrm{BC}$ performance $>\mathrm{XY}$ performance; see Schlichting and Preston, 2014). Voxelwise statistics were calculated using permutation tests implemented in Randomise, part of FSL. Correction for multiple 
comparisons was then performed within a priori HPC and MPFC ROIs using small volume correction implemented in 3dClustSim (Cox, 1996) as described above. We also assessed the relationship between connectivity and AC inference performance within the performance-related regions identified above. Contrast values quantifying the degree of connectivity enhancement with the seed region following overlapping $B C$ encoding were extracted and related to AC performance using partial correlation (controlling for $\mathrm{XY}$ performance).

As the above analysis searches for regions demonstrating a somewhat complex association with behavior (the difference in post-BC and post- $\mathrm{XY}$ connectivity measures relating more to $\mathrm{BC}$ than $\mathrm{XY}$ performance), it is difficult to know the precise underlying pattern that gives rise to this result. To further describe the results of the above analysis, we extracted post-BC and post-XY connectivity measures separately from significant regions identified above (i.e., showing either an overall connectivity enhancement or an enhancement-performance relationship). These measures were compared across two groups of individuals demonstrating different behavioral signatures: those showing behavior consistent with a facilitative effect of prior knowledge on new learning, and those evidencing proactive interference. We used memory for the non-overlapping $X Y$ pairs as a baseline, indexing general memory ability; note that $B C$ and $X Y$ pairs are matched in terms of content type (two objects) and number of learning opportunities (one per pair). Thus, differences in memory for these two types of associations must be related to the presence or absence of prior related knowledge. We reasoned that participants who benefitted from prior knowledge during new encoding-who might learn $B C$ pairs by integrating them into prior $A B$ knowledge-would show better memory 
for the overlapping $B C$ pairs relative to non-overlapping $X Y$ pairs (facilitation group; $B C$ performance $>X Y$ performance, numeric split; $N=15$ ). Other participants, in contrast, might be hindered by the prior $A B$ knowledge, as it interferes with new $B C$ encoding. These participants are expected to show poorer memory for $B C$ relative to $X Y$ pairs (interference group; BC performance $<X Y$ performance, numeric split; $N=16$ ). This analysis excluded individuals who had numerically identical $B C$ and $X Y$ performance ( $N$ = 4). Effects were quantified using $2 \times 2$ mixed ANOVAs with rest scan (post-BC or post-XY) as the within-subjects factor and behavioral signature (facilitation or interference) as the between subjects factor t-tests. The reader should note that these regions were identified precisely because they showed a significant post-BC enhancement or relationship between enhancement and behavior. Thus, it is not the relationship itself but the nature of that relationship among individuals showing these two behavioral signatures that we sought to isolate.

Due to the large and potentially heterogeneous nature of the anatomical MPFC ROI (Ongür and Price, 2000; Price and Drevets, 2009; Roy et al., 2012), we repeated the main rest-based connectivity analysis described above using the MPFC clusters defined in the interaction contrast (depicted in Fig. 2B) as seed regions.

Additionally, to alleviate the possibility that rest-phase connectivity might be attributed to lingering effects of the task itself, we repeated the analysis omitting the first 30 TRs (60 seconds) of the rest scan. This analysis lengthened the delay between study and rest connectivity analyses to roughly 3-4 minutes (in addition to the approximate 2-3 minute delay between scans for image reconstruction and communication with the participant). 


\subsection{Control analyses}

As encoding order was counterbalanced across participants, different individuals experienced differences in time (Schlichting and Preston, 2014) and mnemonic demand between initial $A B$ pair pre-training and overlapping $B C$ study. While one group encoded overlapping $B C$ pairs following $A B$ learning, the other encoded non-overlapping $X Y$ pairs in between learning $A B$ and $B C$. One might predict that these differences in encoding order may impact our neural measures of functional activation or interregional connectivity during study and/or post-encoding rest. For instance, one possibility is that the present findings are driven primarily by the group that encoded $B C$ before $X Y$, as the degree to which $A B$ knowledge is brought to bear during $B C$ learning might decrease as $A B$ experience becomes more remote (i.e., temporal proximity of the related experiences may influence these neural mechanisms). Moreover, there may be behavioral differences across groups. Accordingly, we performed additional control analyses to assess the effects of encoding order on our behavioral and neural measures of interest.

\subsubsection{Effects of encoding order on behavior}

It is possible that the order of encoding overlapping $\mathrm{BC}$ versus non-overlapping $\mathrm{XY}$ associations might differentially promote memory, as these two groups of participants experienced differences in the relative timing of prior $(A B)$ knowledge formation and related (BC) encoding. We tested whether performance differed as a function of encoding order using a $2 \times 2$ mixed ANOVA. Trial type $(B C, X Y)$ was the 
within-subjects factor and encoding order was the between-subjects factor, with performance (proportion correct) serving as the dependent measure. This analysis revealed no significant effect of encoding order (main effect and interaction; both $\mathrm{F}<$ 0.93, both $p>0.344)$, demonstrating that differences in the relative timing had no significant impact on participants' ability to encode the pairs.

\subsubsection{Effects of encoding order at task}

We interrogated the encoding phase to determine whether the degree of taskrelated functional activation or connectivity differed significantly as a function of encoding order. We first focused on clusters showing a significant subsequent recall by prior knowledge condition interaction in univariate activation across the group. We performed a $2 \times 2 \times 2$ mixed ANOVA with subsequent recall and trial type as withinsubjects factors and encoding order as the between-subjects factor. Univariate activation served as the dependent measure. We carried out this analysis for all three regions identified in the main analysis as showing a significant interaction effect within our a priori regions (two MPFC clusters and one HPC cluster; see Fig. 2). We corrected for multiple comparisons in the three ROls using Bonferroni correction, which yielded a critical $p$-value of 0.017 . Encoding order did not significantly affect the observed interaction between subsequent recall and prior knowledge condition in any region (main effects and 3-way interactions; all $F_{1,33}<2.49$, all $p>0.125$ ).

We also determined whether task-phase functional connectivity assessed using PPI differed significantly across our counterbalancing groups. We performed a $2 \times 2$ mixed ANOVA with trial type as the within-subjects factor and encoding order as the 
between-subjects factor. We note that subsequently correct versus incorrect trials were already contrasted within run; thus, the dependent measures in this analysis reflected the connectivity difference for subsequently correct relative to incorrect trials, separately for $\mathrm{BC}$ and $\mathrm{XY}$ conditions. This analysis was performed for all six regions identified previously in the main PPI analyses for FFA, HPC, and MPFC seeds (for a total of four HPC clusters and one MPFC cluster; see Fig. 3). Bonferroni correction across the five ROls yielded a critical p-value of 0.01 . We observed no significant effect of encoding order on connectivity measures in any of these regions (main effects and interactions; all $F_{1,33}<3.27$, all $\left.p>0.08\right)$.

\subsubsection{Effects of encoding order at rest}

Next, we investigated the relationship between functional connectivity during rest and encoding order. We first performed a one-way ANOVA to assess whether the degree of connectivity enhancement following $\mathrm{BC}$ learning differed as a function of encoding order. Neural measures (degree of connectivity enhancement for post-BC versus post-XY rest) were grouped by encoding order. There was no significant effect of encoding order on the degree of connectivity enhancement $\left(F_{1,33}=1.39, p=0.248\right)$.

We performed one-way analyses of covariance (ANCOVA) to determine whether the observed relationships between functional connectivity during rest and performance were related to encoding order. The neural measure served as the independent measure; behavior was the dependent measure. Encoding order was the grouping variable. This analysis was performed for all seven regions identified in the main analyses as relating to performance when FFA, HPC, or MPFC served as the seed 
region (for a total of five HPC clusters and two MPFC clusters; see Figs. 4 and 6). We corrected for multiple comparisons in the seven ROls using Bonferroni correction, yielding a critical $p$-value of 0.007 . There was no significant effect of encoding order on the observed relationship between connectivity and $\mathrm{BC}$ performance controlling for $\mathrm{XY}$ performance at our corrected threshold for any region (main effects and interactions; all $F_{1,33}<5.64$, all $\left.p>0.024\right)$. We note that the one region in which the interaction effect would be considered significant at a more lenient statistical threshold $\left(F_{1,33}=5.64, p=\right.$ 0.024) was the cluster in left anterior HPC when FFA served as the seed. Interestingly, this interaction was driven by a stronger relationship between connectivity and performance among participants who encoded $X Y$ before BC. This is the opposite of what would be predicted by a temporal proximity account, suggesting that our findings are unlikely to reflect merely lingering effects of $A B$ encoding.

\subsection{Structural connectivity}

DTI data analysis was carried out using tools from FDT (FMRIB's Diffusion Toolbox; Behrens et al., 2007) version 3.0 and Tract-Based Spatial Statistics (TBSS; Smith et al., 2006), both part of FSL. We were interested in characterizing how white matter structure in pathways connecting HPC and MPFC related to memory for critical $\mathrm{BC}$ associations relative to control $\mathrm{XY}$ associations. We hypothesized that as the HPCMPFC circuit supports the updating of existing memories with new information, greater white matter integrity in these tracts should relate to superior overlapping BC pair memory, after accounting for overall differences in memory ability (i.e., performance on non-overlapping $X Y$ pairs). 
To test this hypothesis, we first isolated tracts connecting anatomical HPC and MPFC ROls as follows. Registration was carried out within FDT using FLIRT and FNIRT for structural and standard space transformations, respectively. DTI data were first corrected for eddy currents, and then a diffusion tensor model was fit at each voxel of the DTI data for each participant. We generated probability distributions of diffusion parameters at each voxel in the brain for each participant using Bayesian Estimation of Diffusion Parameters Obtained using Sampling Techniques (BEDPOSTX). We then performed tractography between HPC and MPFC ROIs using PROBTRACKX, which uses the voxelwise probability distributions to generate a distribution of pathways connecting specified regions. Probabilistic tractography was carried out for each individual in native DTI space. Each individual's anatomical ROIs were registered to their DTI data using FLIRT transformations computed previously. Tractography was run twice for each participant (number of samples $=5000$, curvature threshold $=0.2$, maximum number of steps $=2000$, step length $=0.5 \mathrm{~mm}$ ): once from HPC to MPFC (waypoint or inclusion mask); and once from MPFC to HPC. In using this approach, we identified tracts that pass through both ROls for each individual.

Each individual's HPC-MPFC and MPFC-HPC connectivity distribution maps were thresholded to exclude voxels through which fewer than $5 \%(250)$ of all sampled pathways passed. They were then added together to create a single bidirectional white matter ROI for each participant. These white matter ROls were warped to MNI space using FNIRT transformations computed previously in FDT. ROls were summed across participants to create a single, group $\mathrm{ROI}$ in standard space encompassing all tracts 
connecting HPC and MPFC. The overlap of white matter ROls across participants is depicted in Fig. 6A.

Fractional anisotropy (FA) was used as our metric of white matter integrity within this ROI. FA quantifies the degree to which water molecules diffuse in a directional (i.e., anisotropic) manner, and tends to be high when white matter fibers are oriented similarly (i.e., when coherence is high). All subjects' FA data were aligned to common space using FNIRT carried out in TBSS. A mean FA image was then generated and thinned to create a mean FA skeleton, which represents the centers of all tracts common to the group. Each subject's aligned FA data was then skeletonized, i.e., projected onto this group skeleton. Mean FA values were extracted from each individual's skeletonized data across all voxels within the group white matter ROI. FA was related to $B C$ memory and $A C$ inference performance across participants controlling for $\mathrm{XY}$ memory using partial correlation (for a similar approach see Schlichting and Preston, 2014).

To assess the specificity of this relationship to HPC-MPFC pathways, we also identified a control white matter ROI, the corticospinal tract, using the Johns Hopkins University (JHU) white-matter tractography atlas (Hua et al., 2008). FA values were then derived from the skeletonized FA data and related to performance measures using partial correlation as described above.

\subsection{Stepwise multiple regression}

Stepwise multiple linear regression analyses were performed to further assess the degree to which rest-phase functional and structural connectivity measures were 
independently related to performance. Mean FA values extracted from the HPC-MPFC tract of interest served as the measure of structural connectivity. For functional connectivity, we averaged across the connectivity measures from all four clusters (two HPC, two MPFC; depicted in Fig. 4A, right and Fig. 4B) identified as significantly relating to performance in the rest-phase analyses seeded with HPC or MPFC. Two sets of stepwise regression models were run: one with $B C$ performance as the dependent variable, and one with $A C$ performance as the dependent variable. $X Y$ performance, functional connectivity, and structural connectivity measures for each participant were entered into the regression stepwise as predictors. Participants were treated as a random effect.

\section{RESULTS}

\subsection{Behavioral performance}

Behavioral performance was as we have published previously (Schlichting and Preston, 2014). Briefly, as intended, $A B$ pairs were well learned by the fourth test block (range: $80 \%-100 \%$, mean \pm SEM: $97.3 \% \pm 0.9 \%$ correct recall). Importantly, as BC $(11.7 \%-$ $86.7 \%, 41.5 \% \pm 3.3 \%$ correct) and $X Y(10 \%-78.3 \%, 42.4 \% \pm 3.4 \%$ correct) pairs were matched on all dimensions except prior knowledge, we were able to directly compare neural engagement and performance in these two conditions (Fig. 1C). Interestingly, we observed no difference between $B C$ and $X Y$ performance across the group $\left(t_{34}=0.40, p\right.$ $=0.693)$; rather, relative performance on these two conditions was highly variable across individuals (see Schlichting and Preston, 2014 for a more in-depth discussion of 
the factors modulating the interfering versus facilitative effects of prior knowledge on new learning). This variability enabled us to investigate how neural processes engaged during task and post-encoding rest periods related to performance for overlapping $B C$ relative to control non-overlapping $X Y$ pairs. We also found that performance on $A C$ inferences $(6.7 \%-83.3 \% ; 41 \% \pm 3.5 \%$ correct) paralleled BC memory (Schlichting and Preston, 2014), suggesting that $A$ items were not forgotten over the course of the experiment.

\subsection{Hippocampus and medial prefrontal cortex are recruited during encoding of overlapping events}

The above framework suggests that HPC and MPFC will be recruited during learning, specifically during episodes in which interference is reduced through memory updating. We predicted greater activation in these regions during encoding of overlapping BC relative to non-overlapping $X Y$ associations. Importantly, we focused on only those associations that were subsequently remembered in both prior knowledge conditions. We searched for this BC correct $>\mathrm{XY}$ correct effect both at the whole brain level and within HPC and MPFC regions of interest (ROIs). Two clusters survived correction for multiple comparisons across the whole brain: one in posterior cingulate $(-3.75,-39.75$, 21.6; cluster extent $\mathrm{k}=230$; all cluster extents reported in functional dimensions, $3.75 \mathrm{x}$ $3.75 \times 3.6 \mathrm{~mm}$ ) and one extending from precuneus on the medial surface to superior

parietal lobule (-7.5, -66, 39.6; $\mathrm{k=608)}$ (Fig. 2A). Small volume correction revealed significant clusters in both left HPC $(-33.75,-13.4,-21.6 ; \mathrm{k}=21)$ and in MPFC (-3.75, 35.25, -21.6; $\mathrm{k}=49$ ) (Fig. 2A). 
A Main effect of prior knowledge

$\mathrm{BC}>\mathrm{XY}$, correct trials only
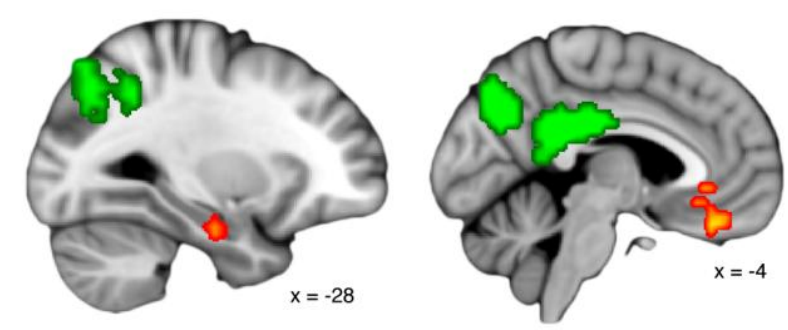

B Recall $\times$ prior knowledge interaction

$\mathrm{BC}$ correct - BC incorrect $>\mathrm{XY}$ correct - $\mathrm{XY}$ incorrect
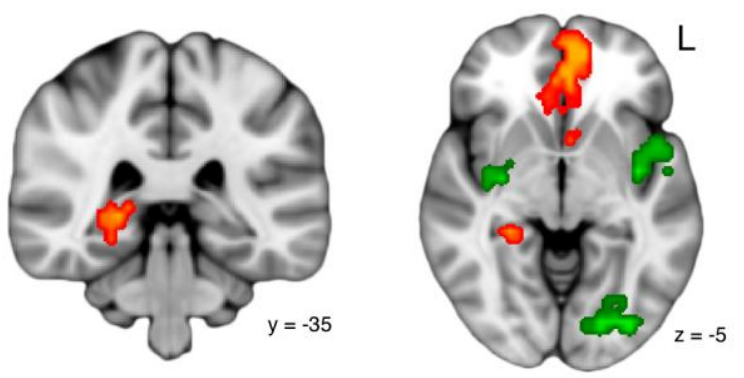

Figure 2. Activation during overlapping encoding predicts memory updating. Maps include clusters significant at the whole-brain level (green) as well as those that survived correction within a priori HPC and MPFC ROls (orange). (A) Main effect of prior knowledge. Regions in parietal cortex and posterior cingulate survived correction for multiple comparisons across the whole brain. Clusters in both HPC (left) and MPFC (right) showed significantly greater activation during encoding of $B C$ relative to $X Y$ pairs (subsequently correct trials only). Clusters are significant after small volume correction within HPC and MPFC, respectively. For all figures, activations were resampled to the $1 \mathrm{~mm} \mathrm{MNI}$ template for visualization. (B) Interaction of subsequent recall and prior knowledge. Clusters in left fusiform and bilateral insula were significant at the whole brain level. Regions in HPC (left) and MPFC (right) showed a significantly greater subsequent recall effect for $\mathrm{BC}$ relative to $\mathrm{XY}$ pairs during encoding. Clusters are significant after small volume correction within HPC and MPFC, respectively. Coordinates are in $\mathbf{m m}$. See also Fig. $\mathbf{S 1}$ for individual participant data.

We next assessed the link between encoding activation and subsequent behavior. Our key prediction is that HPC-MPFC engagement and connectivity will be enhanced when new information is successfully integrated into memory. Thus, engagement should be greater for those overlapping $B C$ associations that are later 
remembered relative to those that are forgotten. Moreover, because BC study events provide the unique opportunity for memory updating via integration, we predicted HPC and MPFC would show a larger subsequent memory effect for BC than XY associations (i.e., a significant interaction between prior knowledge condition and subsequent memory). At the whole brain level, significant clusters were observed in right (41.25, $2.25,28.75 ; \mathrm{k}=74)$ and left insula $(-37.5,-6,0 ; \mathrm{k}=121)$ as well as left fusiform gyrus $(-15$, $-81,-10.75 ; \mathrm{k}=138$ ) (Fig. 2B). We note that due to differences in the spatial normalization approach used between the current study and our previous study (Schlichting and Preston, 2014), the insula regions were not identified in our prior report of this contrast. Using small volume correction, we found one additional significant cluster in right HPC (22.5, -36, 0; $\mathrm{k}=16)$ and two in MPFC (anterior: $-11.25,50.25,0$; $\mathrm{k}=131$; posterior: $-22.5,1.5,-14.4 ; \mathrm{k}=37$ ) that showed the predicted interaction (Fig. 2B). Control analyses verified that the observed interaction between subsequent recall and prior knowledge condition did not differ by order of $B C$ versus $X Y$ encoding in any region. As our key hypotheses relate to integration-specific neural processes and behavior, all subsequent task-based analyses focus on the prior knowledge condition by subsequent memory interaction.

\subsection{Hippocampal-medial prefrontal functional coupling during learning supports memory integration}

While the above-described activation results suggest that both HPC and MPFC are engaged during integration, we were also interested in assessing whether their degree of functional coupling would predict subsequent memory for overlapping but not non- 
overlapping pairs. To examine how functional coupling during new encoding related to the presence or absence of existing knowledge, we performed two psychologicalphysiological interaction (PPI) analyses with anatomically defined HPC and MPFC, respectively, as seed regions. Using this approach, we were able to test our hypothesis that HPC-MPFC connectivity would be modulated on a trial-by-trial basis as a function of subsequent memory and prior knowledge condition. We predicted greater functional coupling during $\mathrm{BC}$ study trials that were later correct relative to those that were incorrect. Moreover, as this effect should be specific to the overlapping study trials, we looked for HPC and MPFC regions that demonstrated this connectivity difference more for $\mathrm{BC}$ relative to $\mathrm{XY}$ trials.

Consistent with our predictions, we found evidence for recruitment of the HPCMPFC circuit during successful encoding of overlapping information. We observed significant activation within MPFC $(0,24,-21.6 ; \mathrm{k}=26)$ when HPC served as the seed (Fig. 3A); and conversely, two significant clusters in HPC (left: -30, -32.25, -7.2; $k=36$; right: $37.5,-24.75,-7.2 ; k=34)$ when MPFC was the seed (Fig. 3B). These effects did not differ significantly by encoding order. Because our anatomical MPFC ROI spanned a large region, we also performed this analysis using the regions identified in the interaction contrast as seeds (i.e., MPFC clusters in Fig. 2B). This analysis yielded similar results (two HPC clusters; left: $-33.75,-32.25,-7.2$; k=38; right: $33.75,-17.25$, $14.4 ; \mathrm{k}=43)$. 

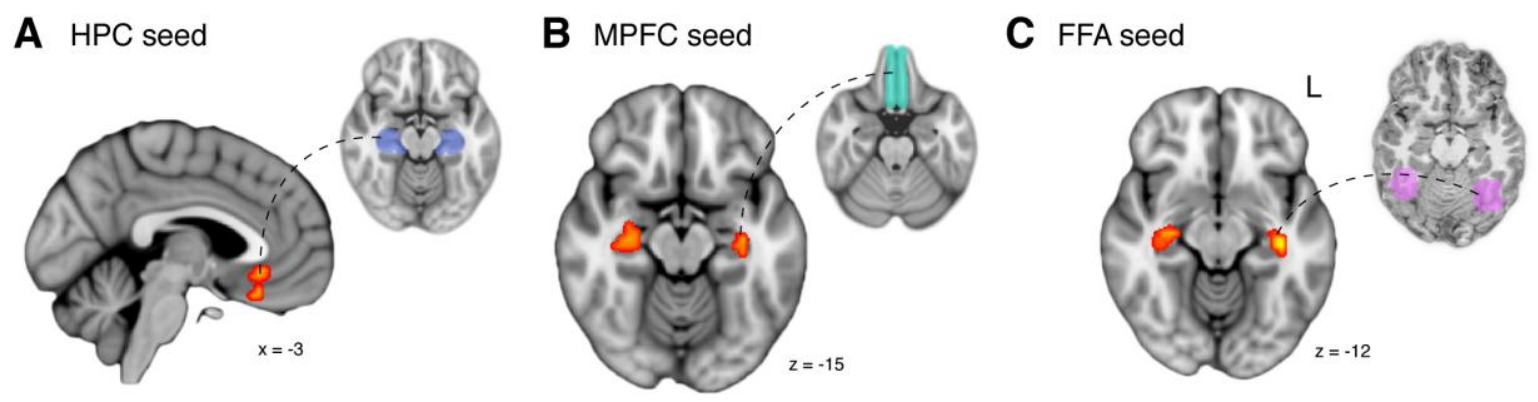

Figure 3. Functional connectivity during overlapping encoding predicts memory updating. Regions for which functional connectivity with MPFC (A), HPC (B), and FFA (C) showed a significantly greater subsequent recall effect for $B C$ relative to $X Y$ pairs during encoding. Clusters are significant after small volume correction within HPC and MPFC. See Fig. S2 for individual participant data.

\subsection{Encoding-phase functional coupling indicates reinstatement of prior} memories in support of updating

A number of existing studies have shown that prior memories may be reactivated during encoding of overlapping experiences (Hupbach et al., 2007; Kuhl et al., 2010; Jones et al., 2012; Zeithamova et al., 2012a; Schlichting and Preston, 2014; Richter et al., 2015). Such reactivation has been linked to better memory for the reactivated content itself (Kuhl et al., 2010), superior learning of the overlapping content (Schlichting and Preston, 2014), and an enhanced ability to make novel judgments that span the two events (Zeithamova et al., 2012a; Richter et al., 2015). Accordingly, we hypothesized that greater functional coupling of HPC and MPFC with face-sensitive visual regions should also be associated with better subsequent memory specifically for the overlapping associations due to the related $A$ stimuli being faces.

To test this prediction, we performed a PPI analysis with each individual's functionally defined bilateral FFA as the seed region. We found significant clusters in bilateral HPC (left: $-37.5,-21,-14.4$; $k=15$; right: 33.75, $-17.25,-14.4 ; \mathrm{k}=15$; Fig. 3C, left). 
There were no significant clusters in MPFC. Results did not differ significantly by encoding order.

\subsection{Degree of rest-phase hippocampal-medial prefrontal connectivity enhancements predicts memory integration performance}

Existing theory suggests that HPC and MPFC interact during sleep to integrate and generalize across discrete experiences (Lewis and Durrant, 2011). Extending these ideas to awake rest periods (Craig et al., 2015b), we hypothesized that greater HPCMPFC connectivity following overlapping encoding would be associated with more memory updating. We interrogated neural engagement following encoding of the overlapping compared with the non-overlapping associations. We hypothesized that we would observe (1) integration-related enhancements in HPC-MPFC connectivity during post-BC encoding rest relative to post- $X Y$ and (2) that the degree of enhancement would relate to behavioral evidence for memory integration across participants.

Using a regression approach, we first searched for voxels in HPC or MPFC whose connectivity with the seed region (MPFC or HPC, respectively) was greater following BC than XY encoding. There were no significant clusters in HPC when MPFC served as the seed. However, when seeding with HPC, we observed a significant region of MPFC that showed the predicted integration-related enhancement $(3.75,16.5,-10.8$; $k=28$; Fig. 4A, left), consistent with the notion that overlapping events trigger a memory updating mechanism. 

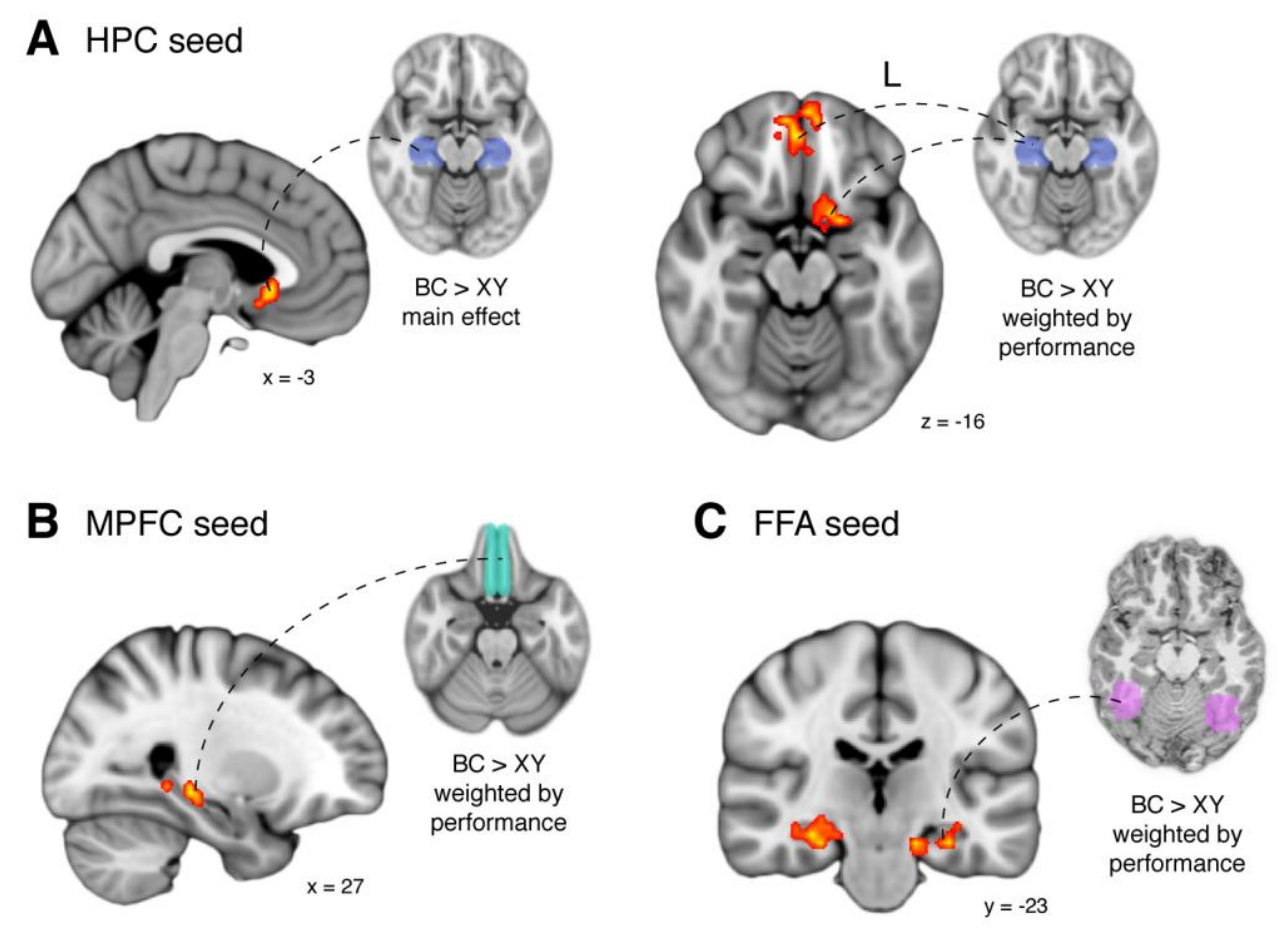

Figure 4. Functional connectivity enhancements during post-encoding rest tracked integration performance. (A) Left, cluster in MPFC showing greater connectivity with HPC following BC than XY encoding. Right, two clusters in MPFC for which the degree of functional connectivity enhancement with HPC during post-BC encoding rest period tracked more with BC than XY performance across participants. (B) HPC clusters for which enhancement tracked more with $B C$ than $X Y$ performance. (C) Functional connectivity with FFA during post-BC encoding rest period tracked more with $\mathrm{BC}$ than $X Y$ performance across participants in HPC. For all panels, clusters are significant after small volume correction within HPC and MPFC. Coordinates are in $\mathrm{mm}$. See also Fig. 5 for effects split by behavioral signature and Fig. S3 for individual participant data.

We also hypothesized that the degree to which participants showed enhanced connectivity following encoding of overlapping relative to non-overlapping associations would correlate with their behavioral evidence for memory integration. That is, if the observed functional connectivity truly reflects integration-specific processes, one would expect that these connectivity enhancements would support performance only for overlapping content. Thus, we identified MPFC and HPC voxels whose connectivity was more predictive of $\mathrm{BC}$ than $\mathrm{XY}$ performance. We found two significant clusters in MPFC 
(anterior: $3.75,50.25,-14.4$; $k=34$; posterior: $-18.75,5.25,-10.8 ; k=19$ ) when HPC served as the seed (Fig. 4A, right). We also identified two clusters in HPC (left: -26.25, 28.5, -7.2, k=31; right: $26.25,-28.5,-7.2, k=24)$ when MPFC served as the seed (Fig. 4B).

We further interrogated the clusters identified as showing the predicted patterns $(B C>X Y$ main effect and relationships to performance, depicted in Fig. 4A-B) to determine how connectivity during post-BC and post-XY rest scans related to the different behavioral signatures exhibited across participants. We did this by dividing our group into individuals showing behavior consistent with a facilitative versus interfering effect of prior $A B$ knowledge on $B C$ learning, with $X Y$ pairs serving as the baseline. We reasoned that participants for whom prior knowledge was beneficial to new learning would show better memory for the overlapping $B C$ relative to their non-overlapping (baseline) XY pair memory (facilitation group; BC performance $>\mathrm{XY}$ performance, numeric split). Conversely, if prior $A B$ knowledge interferes with the learning of new related information, we would expect to see lower memory for $B C$ relative to $X Y$ pairs (interference group; BC performance $<\mathrm{XY}$ performance, numeric split).

For the MPFC cluster showing enhanced connectivity with HPC for BC versus $X Y$ rest across the group (Fig. 4A, left), splitting by behavioral signature revealed that this effect was driven by enhanced connectivity for $B C$ versus $X Y$ among participants demonstrating behavioral facilitation (Fig. 5A, top). Interrogating clusters for which the degree of connectivity enhancement tracked with performance across participants, posterior MPFC (Fig. 4A, right) showed a pattern consistent with memory updating only in the facilitation group, with greater connectivity in that group after $B C$ than $X Y$ 
encoding (Fig. 5A, bottom left). Anterior MPFC (Fig. 4A, right) demonstrated a similar connectivity enhancement following BC learning for the facilitation group, but showed the opposite pattern for the interference group; that is, greater connectivity following $X Y$ than BC encoding (Fig. 5A, bottom right). HPC regions (Fig. 4B) both showed patterns consistent with integration for the facilitation group (Fig. 5B), albeit weakly in right HPC (Fig. 5B, right).

Control analyses revealed that these effects did not differ significantly as a function of encoding order. To limit the possible effects of the immediately preceding study on rest-phase connectivity, we also performed the above analyses excluding the first minute of the rest scan. This analysis yielded similar results (HPC seed, main effect MPFC cluster: $7.5,27.75,-14.4 ; \mathrm{k}=24$; performance-related MPFC cluster 11.25 , 39, 10.8; k=30; MPFC seed, performance-related right HPC cluster: 26.25, $-32.25,0 ; k=29$ ), suggesting that these findings are unlikely to be the result of continuing study-phase engagement.

We also assessed the across-participant relationship between connectivity enhancements in these regions and $A C$ inference performance. As expected, all regions exhibited significant positive associations with inference performance (partial correlations controlling for $X Y$ performance; all $r_{32}>0.49$, all $\left.p<0.003\right)$. Moreover, seeding with MPFC regions defined from the univariate interaction contrast instead of the large anatomical MPFC ROI yielded similar clusters in HPC (left: $-15,-39.75,0$; $\mathrm{k}=22$; right: $33.75,-32.25,-7.2 ; \mathrm{k}=20$ ). These results are consistent with the prediction that enhanced HPC-MPFC functional coupling following overlapping encoding supports 
new learning via the integration of that newly learned content into prior related memories.

\section{A HPC seed}
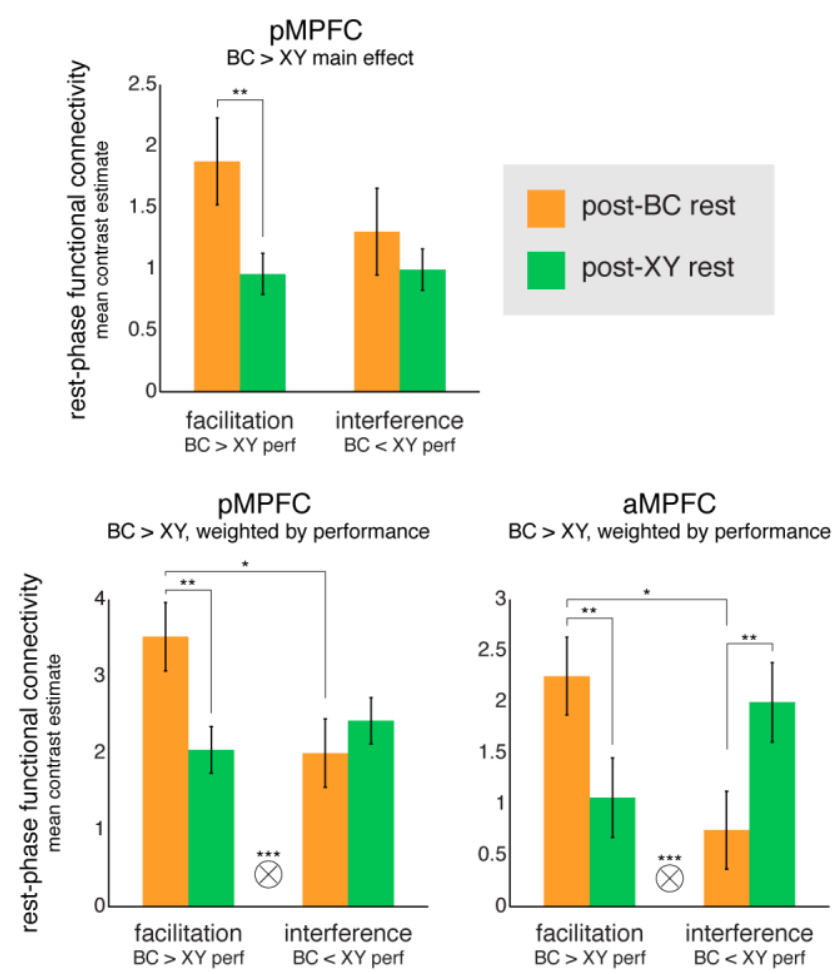

B MPFC seed
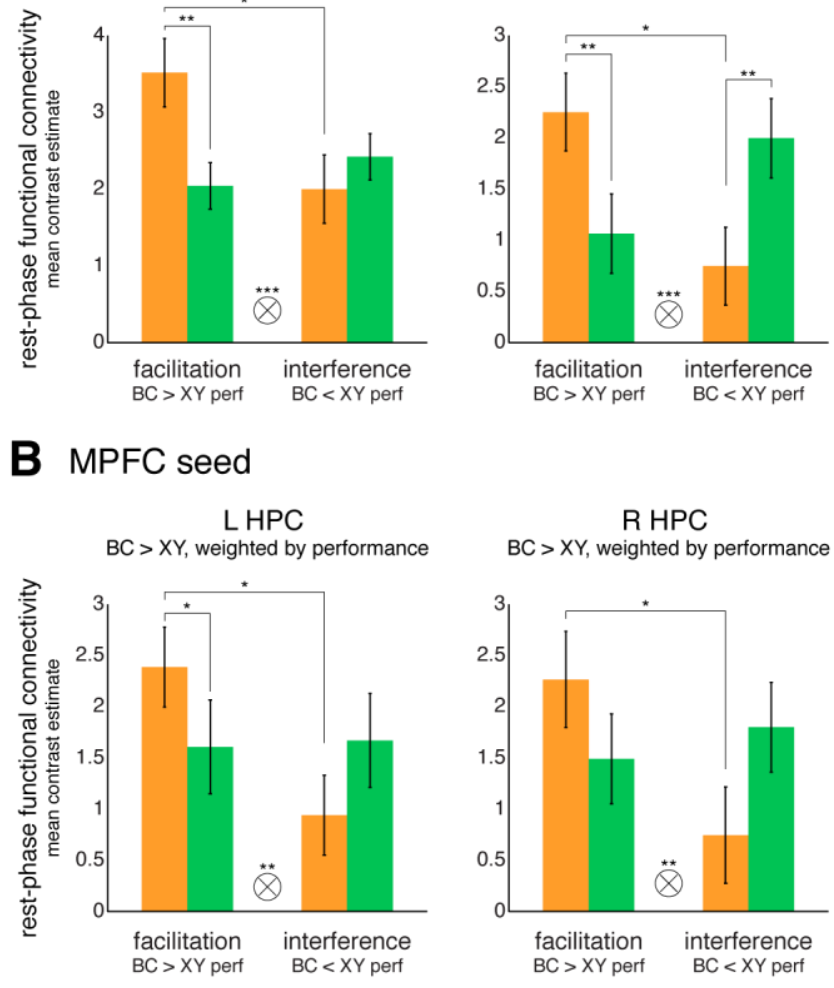
R HPC $\mathrm{BC}>\mathrm{XY}$, weighted by performance

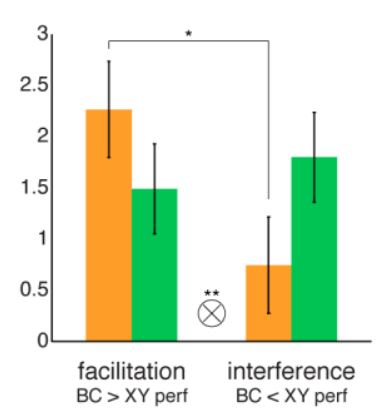

Figure 5. Rest-phase functional connectivity as a function of rest scan and behavioral signature in clusters demonstrating significant enhancement effects (shown in Fig. 4). Regions were defined using (A) HPC and (B) MPFC seeds for showing either enhanced connectivity during the $B C$ relative to $X Y$ rest scan across the group (top chart, cluster depicted in Fig. 4A, left) or a relationship between the degree of $B C>X Y$ enhancement 
and individual differences in performance (bottom four charts, clusters depicted in Figs. 4A, right and $4 B$ ). Connectivity values from the post-BC (orange) and post-XY (green) rest scans were extracted from these clusters and averaged across participants showing facilitation (left bar pairs) and those showing interference (right bar pairs). pMPFC, posterior MPFC; aMPFC, anterior MPFC. Tensor product symbol denotes rest scan by behavioral signature interaction. Asterisks indicate significant level, * $p<0.05$; ${ }^{* *} p<0.01 ;{ }^{* *} p<0.001$.

\subsection{Rest-phase connectivity demonstrates offline processing of related memories}

\section{in support of updating}

A number of studies in both rodents and humans have provided evidence that mnemonic content is processed in the brain during offline periods (e.g., rest and sleep) (Tambini et al., 2010; Jadhav et al., 2012; Deuker et al., 2013; Staresina et al., 2013). For instance, content-specific increases in HPC-neocortical connectivity have been demonstrated following associative encoding (Tambini et al., 2010). Such connectivity enhancements were also related to performance, suggesting that offline processing strengthens memory for recent experiences. We reasoned that immediately following overlapping encoding, HPC and MPFC might show greater connectivity with neocortical regions sensitive to the overlapping content (here, $A_{\text {faces }}$ ), reflecting persistence of the memory updating process. That is, although participants most recently encoded objectobject associations in both $\mathrm{BC}$ and $\mathrm{XY}$ study phases, we predicted that enhanced functional coupling with face-sensitive regions (FFA) during post-BC relative to post-XY would be associated with superior performance.

Accordingly, we performed a voxelwise regression analysis with FFA serving as the seed region. We found three significant clusters within HPC for which the degree of connectivity enhancement during the post-BC encoding rest period related more to $\mathrm{BC}$ than $X Y$ performance (Fig. 4C): one in right $(30,-21,-14.4 ; k=55)$ and two in left 
(anterior: $-11.25,-9.75,-21.6$; $k=14$; posterior: $-15,-36,0 ; k=35$ ) HPC. Connectivity within these HPC clusters was also significantly related to AC inference performance (partial correlations controlling for $X Y$ performance; all $r_{32}>0.44$, all $p<0.009$ ). There were no significant clusters in MPFC. These findings did not differ by encoding order. Similar results were obtained after omitting the first minute of the rest scan (right HPC: 18.75, -24.75, -14.4, k=70; left HPC: -22.5, -39.75, 3.6, k=29; MPFC: 3.75, 5.25, -10.8, $k=35)$.

\subsection{White matter integrity predicts performance in tracts connecting HPC and MPFC}

The preceding findings indicate that HPC-MPFC activation and functional coupling predicts subsequent memory updating, both during learning on a trial-by-trial basis and during offline periods across participants. We hypothesized that the structural integrity of the white matter tracts connecting these regions would also predict individual differences in the ability to learn overlapping relative to non-overlapping associations. Here, we define white matter integrity as fractional anisotropy (FA), a commonly used measure quantifying the degree to which water molecules diffuse in a directional manner. High FA suggests high white matter integrity or tract coherence. There was no relationship between FA in our control (corticospinal) tract and behavior (i.e., partial correlation with $B C$ and $A C$ performance controlling for $X Y$; $B C: r_{22}=0.29, p=0.169$; $\left.A C: r_{22}=0.28, p=0.178\right)$. However, we did find a positive relationship between FA in tracts connecting HPC and MPFC (Fig. 6A) and both BC pair memory and AC inference, after statistically controlling for individual differences in general memory ability (i.e., 
partial correlation with $B C$ and $A C$ performance controlling for $X Y$; $B C: r_{22}=0.42, p=$ 0.039, Fig. 6B; $A C: r_{22}=0.45, p=0.028$.
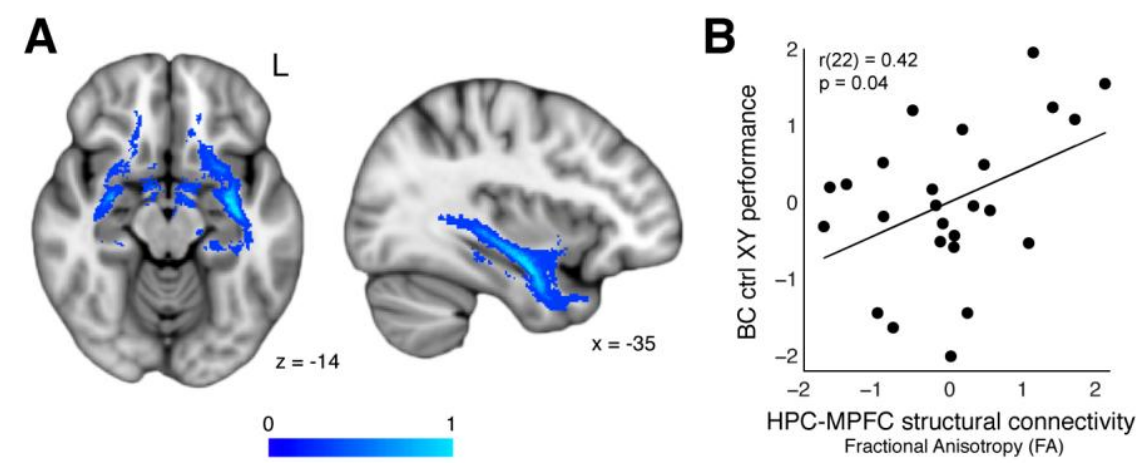

Figure 6. HPC-MPFC structural connectivity predicts memory updating across participants. (A) Group map depicting overlap across participants of tracts connecting HPC-MPFC. Tracts were determined for each individual using probabilistic tractography, warped to the $\mathrm{MNI}$ template and combined across participants. Colorbar indicates the proportion of participants with tracts in each voxel. (B) Mean fractional anisotropy (FA) values were extracted for each participant within the group $\mathrm{ROI}$ depicted in panel $A$. Scatterplot shows across-participant relationship between FA within HPC-MPFC white matter tracts and $\mathrm{BC}$ performance, plotted as $\mathrm{Z}$-scored residuals after regressing both on $\mathrm{XY}$ performance.

\subsection{Structural and rest-phase functional HPC-MPFC connectivity independently explain variance in integration performance}

To investigate the degree to which HPC-MPFC structural and rest-phase functional connectivity measures independently explained variance in subsequent learning, we next performed two stepwise multiple linear regression analyses with (1) XY performance and indices of (2) structural and (3) rest-phase functional connectivity as independent variables; and $\mathrm{BC}$ and $\mathrm{AC}$ performance, respectively, as the dependent variables. For both $\mathrm{BC}$ and $\mathrm{AC}$ performance, the prediction models included all three independent variables. For BC performance, the full model fit was statistically significant $\left(\mathrm{F}_{3,21}=64.379, \mathrm{p}<1 \times 10^{-9}\right)$, accounting for $88.8 \%$ of the variance in $\mathrm{BC}$ performance 
(adjusted $R^{2}$ ) and was superior to models with just one (adjusted $R^{2}=0.589$ ) or two (adjusted $R^{2}=0.862$ ) predictors. All three independent variables showed a significant positive relationship to $B C$ performance $\left(X Y\right.$ performance: $\beta=0.92, p<1 \times 10^{-10}$; functional connectivity: $\beta=0.50, p<1 \times 10^{-6}$; structural connectivity: $\beta=0.17, p=0.022$; all statistics reflect standardized $\beta$ ), demonstrating the unique contributions of structural and functional HPC-MPFC connectivity to subsequent learning of related experiences. Similar results were found in the stepwise regression model predicting AC performance (full model fit $F_{3,21}=46.547, p<1 \times 10^{-8}$; adjusted $R^{2}=0.851$, increased from 0.565 and 0.815 with one or two predictors, respectively; $X Y$ performance: $\beta=0.90, p<1 \times 10^{-9}$; functional connectivity: $\beta=0.48, p<1 \times 10^{-4}$; structural connectivity: $\beta=0.20, p=$ $0.021)$.

\section{DISCUSSION}

The present study provides convergent evidence from functional and structural MRI methodologies that HPC-MPFC interactions promote integration of new content into existing knowledge, both at the level of individual memories and across participants. This work is consistent with the idea that MPFC resolves competition among memories through integration (Preston and Eichenbaum, 2013) and promotes generalization across episodes (Schnider, 2003; Gilboa et al., 2006; Koscik and Tranel, 2012; Warren et al., 2014). We show that recruitment of the HPC-MPFC circuit benefits new learning, providing a neurobiological account of the age-old observation that knowledge can promote the formation of new, related memories (Bartlett, 1932). Integration-related signatures were observed in posterior MPFC, consistent with this region's proposed role 
in integrating across inputs (Nieuwenhuis and Takashima, 2011). Structural measures also predicted performance, with greater tract coherence between HPC and MPFC being associated with superior memory integration. We further extend prior human and animal work by showing that content-specific cortical modules interacted with this circuit in service of integration. Specifically, we found that FFA was functionally connected to HPC when new content related to previously learned face-object associations. Despite the large individual differences in behavior in our study, follow-up analyses ensured that our observed effects were not driven by only a few high-performing participants (see Figs. S1-S3).

Enhanced HPC-MPFC coupling when new learning related to prior knowledge was observed not only during intentional study, but also during post-encoding rest periods. Despite the fact that immediately preceding experiences consisted of objectobject associations in both cases, functional connectivity and its relationship to memory performance differentiated post-BC from post- $\mathrm{XY}$ encoding rest periods. We found that enhanced HPC functional coupling with posterior MPFC following overlapping encoding was associated with behavioral evidence for superior integration. This work extends theories on the sleep-based mechanisms thought to support memory reorganization and integration (Lewis and Durrant, 2011) by providing an empirical test during awake rest. We also observed enhanced HPC functional coupling with FFA during this period, suggesting processing of integrated traces incorporating more remotely encoded but related content $\left(A_{\text {face }}\right.$ items). Taken together, these results suggest a behavioral benefit (Craig et al., 2015b) to offline processing of integrated memories on subsequent recall of the newly learned information. 
Theories propose that HPC and MPFC form a highly interactive and dynamic circuit impacting both encoding and retrieval. In particular, MPFC is thought to form memory models (Schacter et al., 2012; St. Jacques et al., 2013) that bias HPC retrieval toward task-relevant memories (Kroes and Fernández, 2012; van Kesteren et al., 2012; Preston and Eichenbaum, 2013). When new content overlaps with existing knowledge, MPFC memory models may thus be activated, guiding HPC retrieval of relevant knowledge. HPC encoding mechanisms would then bind current experience to the reactivated content to form integrated memory traces (Preston and Eichenbaum, 2013; Schlichting and Preston, 2015), updating memory models (van Kesteren et al., 2012) through projections to MPFC.

The present findings build upon these theories to provide an understanding of the roles played by subregions of HPC (Giovanello et al., 2009; Poppenk et al., 2013) and MPFC (Ongür and Price, 2000; Nieuwenhuis and Takashima, 2011; Roy et al., 2012; Schlichting et al., 2015) in memory integration. For instance, univariate analyses revealed that activation in posterior HPC predicted subsequent memory for overlapping associations. As previous work has implicated posterior HPC in representing specific event elements (Komorowski et al., 2009; Liang et al., 2012; Poppenk et al., 2013; Preston and Eichenbaum, 2013; Schlichting et al., 2015), one possibility is that posterior HPC drives retrieval of mnemonic details during new $B C$ encoding.

Notably, in contrast to the univariate activation observed in posterior HPC, taskphase connectivity with MPFC was found more anteriorly. In addition to having direct anatomical projections to MPFC (Barbas and Blatt, 1995), anterior HPC has been implicated in forming generalized representations that span events (Komorowski et al., 
2009; Liang et al., 2012; Poppenk et al., 2013; Preston and Eichenbaum, 2013). Thus, one possible interpretation of this finding is that detailed memories reactivated by posterior HPC are communicated to anterior HPC, which in turn integrates and transfers them to MPFC. This interpretation is consistent with recent fMRI work showing integration of related memories in anterior HPC and posterior MPFC (see below; Schlichting et al., 2015), with similar neural representations for indirectly related A and C items formed in these regions. Offline processing of integrated traces may occur through coordinated HPC-MPFC interactions during rest, with reactivation of episodic detail (posterior HPC) and integration across episodes (anterior HPC) occurring simultaneously following overlapping encoding.

In the present study, we observed effects primarily in posterior aspects of MPFC (i.e., subgenual MPFC/anterior cingulate cortex $[A C C]$ ) during both encoding and rest. These findings are consistent with work suggesting that this subregion in particular carries out the integrative functions of MPFC (Nieuwenhuis and Takashima, 2011; Roy et al., 2012; Schlichting et al., 2015). Subgenual MPFC exhibits a widespread pattern of anatomical connectivity (Barbas and Blatt, 1995; Cavada et al., 2000; Ongür and Price, 2000), allowing it to integrate across limbic inputs during learning. Damage to this region results in reduced false memory formation (Warren et al., 2014) and poor schema representation (Ghosh et al., 2014), suggesting its involvement in integrating across sources of information. Engagement of subgenual MPFC also increases with consolidation (Nieuwenhuis and Takashima, 2011), perhaps tracking the increasing importance of abstracted neocortical representations as experiences become more remote. The present work extends these ideas to suggest that enhanced engagement 
and connectivity of subgenual MPFC with HPC promotes integration of overlapping events into prior memories (Schlichting et al., 2015). The present results may thus reflect both learning-phase biasing of HPC retrieval mechanisms toward relevant memories and rest-phase processing of integrated memories.

We also observed performance-related connectivity of anterior aspects of MPFC (i.e., rostromedial PFC) with HPC during rest. Interestingly, this region showed a different pattern of connectivity than did subgenual MPFC. In anterior MPFC, connectivity was enhanced during post-BC relative to post- $X Y$ rest for individuals who showed superior $B C$ relative to $X Y$ learning, while the opposite pattern was observed for individuals showing better memory for $X Y$ than $B C$ pairs (Fig. 5A, bottom right). One speculative interpretation of this finding is that while subgenual MPFC integrates overlapping content, anterior MPFC performs a more general mnemonic function. Recent work has demonstrated that this region maintains distinct representations of indirectly related $A$ and $C$ items (Schlichting et al., 2015), consistent with the notion that anterior MPFC stores related memories separately. This region has also been implicated in episodic simulation and future thinking (Okuda et al., 2003; Addis et al., 2009), which require retrieval and restructuring of episodic details. Thus, one possibility is that during post-encoding rest, anterior MPFC guides reinstatement of both overlapping and non-overlapping associations. Enhanced rest-phase anterior MPFCHPC connectivity may therefore be associated with superior memory for the preceding pairs, regardless of their relationship to prior knowledge.

Alternate accounts of the present findings might suggest that enhanced HPC connectivity with prefrontal regions during learning reflects other operations such as 
inhibition (Aron et al., 2014) or retrieval (Schedlbauer et al., 2014; King et al., 2015) of the related $A$ item (without integration). With regards to an interpretation based on inhibition, it is worth noting that such functions have typically been ascribed to right lateral PFC; as such, it is unclear whether MPFC would be expected to perform a similar operation. Moreover, inhibiting the $A$ item during BC encoding would predict forgetting of A's associated with remembered BC pairs due to weakening of the successfully inhibited $A B$ memories. Our behavioral data are not consistent with this interpretation; rather, $\mathrm{BC}$ memory was paralleled by performance on the $\mathrm{AC}$ inference test, which required retrieval of the $A$ items. Thus, our findings suggest that $A B$ pair memories were not weakened by $\mathrm{BC}$ encoding, which conflicts with an inhibition account.

A second possibility is that HPC-MPFC connectivity reflects retrieval of the A item itself, and not necessarily integration across $A B$ and $B C$ events. Under this hypothesis, MPFC may bias HPC to reinstate the prior memory, but the BC memory is formed separately. We suggest that while such an interpretation is a plausible account of our data, it is important to consider several nuances of how this hypothesis relates to the present analyses. As our task-phase connectivity results index the interaction between trial type and subsequent memory, it must be the case that $A$ item retrieval via HPC-MPFC interactions promotes $B C$ encoding. Conflict between prior $(A B)$ retrieved memories and current $(\mathrm{BC})$ experience may be resolved in one of two ways: (1) differentiation, in which the new $B C$ memory is kept distinct from $A B$ (Hulbert and Norman, 2014) or (2) integration of the related $A B$ and $B C$ experiences. We suggest that the particular mechanism reflected in HPC-MPFC connectivity may differ across 
individuals and across subregions, with both mechanisms (Kumaran, 2012; Zeithamova et al., 2012b; Schlichting and Preston, 2015) occurring simultaneously to support inference (Schlichting et al., 2015). As described previously, the present data suggest that while anterior HPC and posterior MPFC may integrate across related memories, posterior HPC and anterior MPFC keep them separate. Interestingly, these results accord with our prior work showing differences in integration and separation of overlapping memories across the anterior-posterior axes of these regions (Schlichting et al., 2015).

Notably, the present findings contrast with a prior study that reported decreased functional coupling for participants with strong prior knowledge (van Kesteren et al., 2010a). We propose that there are at least three factors that might account for these discrepancies. First, while the present study trained both initial and overlapping associations within a single day, the previous study (van Kesteren et al., 2010a) imposed a 24-hour delay between initial learning and overlapping encoding. This difference could have significant implications for memory processes, given the demonstrated importance of sleep for integrating and generalizing across experiences (Buckner, 2010; Lewis and Durrant, 2011). Second, the degree of compatibility between existing knowledge and new experiences—-thought to impact integration demands (van Kesteren et al., 2012)-is difficult to compare across paradigms. Prior work (van Kesteren et al., 2010a) has theorized that integration demands may be heightened when encoding in the context of incompatible related memories, while our paradigm may encourage resolving incompatibilities via integration precisely when strong prior knowledge exists (Zeithamova et al., 2012a; Preston and Eichenbaum, 2013; Bein et al., 
2014). Third, previous work (van Kesteren et al., 2010a) used a large, heterogeneous MPFC ROI to assess connectivity with HPC, thus making it impossible to determine which subregion might have driven their results. Here, we use a voxelwise approach to demonstrate that integration-related connectivity enhancements were found only in posterior MPFC.

We also interrogated white matter structure to find that fractional anisotropy in HPC-MPFC white matter pathways related to memory integration behavior. Prior work (Gerraty et al., 2014) has shown individual differences in resting-state functional connectivity relating to memory integration processes that support generalization. In apparent contrast to the present findings, that study found that intrinsic (i.e., not taskevoked) HPC-MPFC functional connectivity was negatively correlated with behavioral evidence for memory integration (Gerraty et al., 2014). However, HPC and MPFC also showed opposite connectivity-performance relationships with the default mode network (DMN), with low HPC-DMN and high MPFC-DMN connectivity being associated with superior integration. Thus, it is unclear whether the negative relationship between intrinsic HPC-MPFC connectivity and behavior implies more or less reliance on these structures during integration itself. Our results complement this prior report in suggesting that even stable individual differences in structure and function of HPCMPFC circuitry relate to complex memory behaviors. Importantly, our results rely on an unrelated measure of structural connectivity, highlighting the conceptual convergence across methodologies.

While we focus the present report on our a priori HPC and MPFC ROls, it is notable that a number of regions were more engaged during the encoding of 
overlapping $(\mathrm{BC})$ relative to non-overlapping $(\mathrm{XY})$ associations in terms of univariate activation at our whole-brain corrected threshold (Fig. 2). Note that these effects are stronger than those in HPC and MPFC, which did not reach significance at the wholebrain level. One possible reason why a more lenient small volume corrected threshold was necessary to reveal the involvement of HPC and MPFC is signal loss in these regions, due to their proximity to air-tissue interfaces. At the whole brain level, clusters in posterior cingulate and precuneus/superior parietal showed greater engagement for subsequently correct $B C$ versus $X Y$ encoding trials, perhaps reflecting increased demands on top-down attention (Corbetta and Shulman, 2002; Hutchinson et al., 2014) during overlapping encoding. This could include increased attention to either (a) the BC pair presentation itself, which serves as a retrieval cue for $A$ or (b) the contents of retrieval (i.e., allocating attention to the internal representation of A). We also observed activation in left fusiform gyrus and bilateral insula that was more predictive of subsequent memory for $\mathrm{BC}$ than $\mathrm{XY}$ associations, consistent with the idea that engaging these regions during learning promoted memory specifically for the pairs overlapping with prior $A B$ knowledge. One interpretation of the fusiform finding is that activation of this region reflects reinstatement of face information during successful encoding of BC pairs (Schlichting and Preston, 2014), which would be required for successful integration. With regards to insula, it is noteworthy that while cognitive operations like switching and conflict resolution have most often been associated with anterior insula (Menon and Uddin, 2010; Chang et al., 2012), our clusters spanned both posterior and anterior regions. While we do not have a definitive explanation as to why our task would additionally engage posterior insula, it is noteworthy that this subregion 
shows the greatest intrinsic functional coupling with ventral MPFC (Chang et al., 2012). Thus, one speculative interpretation of this finding is that engagement of posterior insula reflects its functional relationship to MPFC, with anterior insula additionally aiding in integrating across internal $(A B)$ and external $(B C)$ sources of information (Chang et al., 2012).

\section{CONCLUSIONS}

We demonstrate that fluctuations in HPC-MPFC connectivity track integration demands on a trial-by-trial basis. Our findings also provide novel insight into the timecourse of integration, suggesting the importance of post-encoding rest periods for offline reorganization of overlapping memories. Evidence of rest-phase integration was specific to posterior MPFC, consistent with the notion of dissociable mnemonic functions across MFPC. Moreover, we demonstrate how underlying HPC-MPFC structure relates to integration ability, providing insight into why some individuals are better able to integrate knowledge than others.

\section{ACKNOWLEDGEMENTS}

The authors thank Jackson Liang, Michael Mack, Tammy Tran, Amelia Wattenberger and Dagmar Zeithamova for assistance with data collection and helpful discussions. This work was supported by the National Institute of Mental Health of the National Institutes of Health under award number R01MH100121 and by the National Science Foundation under CAREER award number 1056019 to ARP; and by the Department of Defense (DoD) through the National Defense Science \& Engineering Graduate 
Fellowship (NDSEG) Program to MLS. The authors declare no competing financial interests. 


\section{REFERENCES}

Addis DR, Pan L, Vu M-A, Laiser N, Schacter DL (2009) Constructive episodic simulation of the future and the past: Distinct subsystems of a core brain network mediate imagining and remembering. Neuropsychologia 47:2222-2238.

Aron AR, Robbins TW, Poldrack RA (2014) Inhibition and the right inferior frontal cortex: One decade on. Trends Cogn Sci 18:177-185.

Avants BB, Tustison NJ, Song G, Cook PA, Klein A, Gee JC (2011) A reproducible evaluation of ANTs similarity metric performance in brain image registration. Neuroimage 54:2033-2044.

Axmacher N, Elger CE, Fell J (2008) Ripples in the medial temporal lobe are relevant for human memory consolidation. Brain 131:1806-1817.

Barbas H, Blatt GJ (1995) Topographically Specific Hippocampal Projections Target Functionally Distinct Prefrontal Areas in the Rhesus Monkey. Hippocampus 5:511533.

Bartlett F (1932) Remembering: A Study in Experimental and Social Psychology. Cambridge: Cambridge University Press.

Behrens TEJ, Berg HJ, Jbabdi S, Rushworth MFS, Woolrich MW (2007) Probabilistic diffusion tractography with multiple fibre orientations: What can we gain? Neuroimage 34:144-155.

Bein O, Reggev N, Maril A (2014) Prior knowledge influences on hippocampus and medial prefrontal cortex interactions in subsequent memory. Neuropsychologia 64:320-330.

Buckner RL (2010) The role of the hippocampus in prediction and imagination. Annu Rev Psychol 61:27-48.

Buzsaki G, Leung LWS, Vanderwolf CH (1983) Cellular bases of hippocampal EEG in the behaving rat. Brain Res Rev 6:139-171.

Cavada C, Compañy T, Tejedor J, Cruz-Rizzolo RJ, Reinoso-Suárez F (2000) The anatomical connections of the macaque monkey orbitofrontal cortex. A review. Cereb Cortex 10:220-242.

Chang L, Yarkoni T, Khaw M, Sanfey A (2012) Decoding the role of the insula in human cognition: functional parcellation and large-scale reverse inference. Cereb Cortex.

Corbetta M, Shulman GL (2002) Control of goal-directed and stimulus-driven attention 
in the brain. Nat Rev Neurosci 3:201-215.

Cornwell BR, Overstreet C, Grillon C (2014) Spontaneous fast gamma activity in the septal hippocampal region correlates with spatial learning in humans. Behav Brain Res 261:258-264.

Coutanche MN, Gianessi CA, Chanales AJH, Willison KW, Thompson-Schill SL (2013) The role of sleep in forming a memory representation of a two-dimensional space. Hippocampus 23:1189-1197.

Cowan N (2004) Verbal recall in amnesiacs under conditions of diminished retroactive interference. Brain 127:825-834.

Cox RW (1996) AFNI: software for analysis and visualization of functional magnetic resonance neuroimages. Comput Biomed Res 29:162-173.

Craig M, Dewar M, Della Sala S, Wolbers T (2015a) Rest boosts the long-term retention of spatial associative and temporal order information. Hippocampus 1027:1-11.

Craig M, Dewar M, Harris MA, Sala S Della (2015b) Wakeful Rest Promotes the Integration of Spatial Memories into Accurate Cognitive Maps. Hippocampus.

Desikan RS, Ségonne F, Fischl B, Quinn BT, Dickerson BC, Blacker D, Buckner RL, Dale AM, Maguire RP, Hyman BT, Albert MS, Killiany RJ (2006) An automated labeling system for subdividing the human cerebral cortex on MRI scans into gyral based regions of interest. Neuroimage 31:968-980.

Deuker L, Olligs J, Fell J, Kranz TA, Mormann F, Montag C, Reuter M, Elger CE, Axmacher N (2013) Memory consolidation by replay of stimulus-specific neural activity. J Neurosci 33:19373-19383.

Dewar M, Alber J, Butler C, Cowan N, Della Sala S (2012) Brief wakeful resting boosts new memories over the long term. Psychol Sci 23:955-960.

Diekelmann S, Born J (2010) The memory function of sleep. Nat Rev Neurosci 11:114126.

Ego-Stengel V, Wilson MA (2010) Disruption of ripple-associated hippocampal activity during rest impairs spatial learning in the rat. Hippocampus 20:1-10.

Eichenbaum H, Dudchenko PA, Wood E, Shapiro M, Tanila H (1999) The hippocampus, memory, and place cells: is it spatial memory or a memory space? Neuron 23:209226.

Ellenbogen JM, Hu PT, Payne JD, Titone D, Walker MP (2007) Human relational memory requires time and sleep. Proc Natl Acad Sci U S A 104:7723-7728. 
Foster DJ, Wilson MA (2006) Reverse replay of behavioural sequences in hippocampal place cells during the awake state. Nature 440:680-683.

Fox MD, Snyder AZ, Vincent JL, Corbetta M, Van Essen DC, Raichle ME (2005) The human brain is intrinsically organized into dynamic, anticorrelated functional networks. Proc Natl Acad Sci U S A 102:9673-9678.

Friston KJ, Buechel C, Fink GR, Morris J, Rolls E, Dolan RJ (1997) Psychophysiological and modulatory interactions in neuroimaging. Neuroimage 6:218-229.

Gerraty RT, Davidow JY, Wimmer GE, Kahn I, Shohamy D (2014) Transfer of learning relates to intrinsic connectivity between hippocampus, ventromedial prefrontal cortex, and large-scale networks. J Neurosci 34:11297-11303.

Gershman SJ, Schapiro AC, Hupbach A, Norman KA (2013) Neural context reinstatement predicts memory misattribution. J Neurosci 33:8590-8595.

Ghosh VE, Gilboa A (2013) What is a memory schema? A historical perspective on current neuroscience literature. Neuropsychologia:1-11.

Ghosh VE, Moscovitch M, Melo Colella B, Gilboa A (2014) Schema Representation in Patients with Ventromedial PFC Lesions. J Neurosci 34:12057-12070.

Gilboa A, Alain C, Stuss DT, Melo B, Miller S, Moscovitch M (2006) Mechanisms of spontaneous confabulations: a strategic retrieval account. Brain 129:1399-1414.

Giovanello KS, Schnyer DM, Verfaellie M (2009) Distinct hippocampal regions make unique contributions to relational memory. Hippocampus 19:111-117.

Gupta AS, van der Meer MAA, Touretzky DS, Redish AD (2010) Hippocampal replay is not a simple function of experience. Neuron 65:695-705.

Hua K, Zhang J, Wakana S, Jiang H, Li X, Reich DS, Calabresi PA, Pekar JJ, van ZijI PCM, Mori S (2008) Tract probability maps in stereotaxic spaces: Analyses of white matter anatomy and tract-specific quantification. Neuroimage 39:336-347.

Hulbert JC, Norman KA (2014) Neural Differentiation Tracks Improved Recall of Competing Memories Following Interleaved Study and Retrieval Practice. Cereb Cortex:1-15.

Hupbach A, Gomez R, Hardt O, Nadel L (2007) Reconsolidation of episodic memories: A subtle reminder triggers integration of new information. Learn Mem 14:47-53.

Hutchinson JB, Uncapher MR, Weiner KS, Bressler DW, Silver MA, Preston AR, Wagner AD (2014) Functional heterogeneity in posterior parietal cortex across attention and episodic memory retrieval. Cereb Cortex 24:49-66. 
Jadhav SP, Kemere C, German PW, Frank LM (2012) Awake Hippocampal SharpWave Ripples Support Spatial Memory. Science 336:1454-1457.

Jones B, Bukoski E, Nadel L, Fellous J-M (2012) Remaking memories: Reconsolidation updates positively motivated spatial memory in rats. Learn Mem 19:91-98.

Karlsson MP, Frank LM (2009) Awake replay of remote experiences in the hippocampus. Nat Neurosci 12:913-918.

King DR, de Chastelaine M, Elward RL, Wang TH, Rugg MD (2015) RecollectionRelated Increases in Functional Connectivity Predict Individual Differences in Memory Accuracy. J Neurosci 35:1763-1772.

Komorowski RW, Manns JR, Eichenbaum H (2009) Robust conjunctive item-place coding by hippocampal neurons parallels learning what happens where. J Neurosci 29:9918-9929.

Koscik TR, Tranel D (2012) The human ventromedial prefrontal cortex is critical for transitive inference. J Cogn Neurosci 24:1191-1204.

Kroes MCW, Fernández G (2012) Dynamic neural systems enable adaptive, flexible memories. Neurosci Biobehav Rev 36:1646-1666.

Kuhl BA, Bainbridge WA, Chun MM (2012) Neural reactivation reveals mechanisms for updating memory. J Neurosci 32:3453-3461.

Kuhl BA, Rissman J, Chun MM, Wagner AD (2011) Fidelity of neural reactivation reveals competition between memories. Proc Natl Acad Sci U S A 108:5903-5908.

Kuhl BA, Shah AT, DuBrow S, Wagner AD (2010) Resistance to forgetting associated with hippocampus-mediated reactivation during new learning. Nat Neurosci 13:501-506.

Kumaran D (2012) What representations and computations underpin the contribution of the hippocampus to generalization and inference? Front Hum Neurosci 6:157.

Kumaran D, Summerfield JJ, Hassabis D, Maguire EA (2009) Tracking the emergence of conceptual knowledge during human decision making. Neuron 63:889-901.

Lewis PA, Durrant SJ (2011) Overlapping memory replay during sleep builds cognitive schemata. Trends Cogn Sci 15:343-351.

Liang JC, Wagner AD, Preston AR (2012) Content representation in the human medial temporal lobe. Cereb Cortex 23:80-96.

Loftus EF (2005) Planting misinformation in the human mind: a 30-year investigation of the malleability of memory. Learn Mem 12:361-366. 
McClelland JL, McNaughton BL, O'Reilly RC (1995) Why there are complementary learning systems in the hippocampus and neocortex: Insights from the successes and failures of connectionist models of learning and memory. Psychol Rev 102:419-457.

Melton AW (1970) The situation with respect to the spacing of repetitions and memory. J Verbal Learning Verbal Behav 9:596-606.

Menon V, Uddin LQ (2010) Saliency, switching, attention and control: a network model of insula function. Brain Struct Funct:1-13.

Moscovitch M, Rosenbaum RS, Gilboa A, Addis DR, Westmacott R, Grady C, McAndrews MP, Levine B, Black S, Winocur G, Nadel L (2005) Functional neuroanatomy of remote episodic, semantic and spatial memory: a unified account based on multiple trace theory. J Anat 207:35-66.

Nadel L, Hupbach A, Gomez R, Newman-Smith K (2012) Memory formation, consolidation and transformation. Neurosci Biobehav Rev 36:1640-1645.

Nader K, Einarsson EÖ (2010) Memory reconsolidation: An update. Ann N Y Acad Sci $1191: 27-41$.

Nader K, Schafe GE, Doux JE Le (2000a) Fear memories require protein synthesis in the amygdala for reconsolidation after retrieval. Nature 406:722-726.

Nader K, Schafe GE, LeDoux JE (2000b) The labile nature of consolidation theory. Nat Rev Neurosci 1:216-219.

Nieuwenhuis ILC, Takashima A (2011) The role of the ventromedial prefrontal cortex in memory consolidation. Behav Brain Res 218:325-334.

O'Reilly JX, Woolrich MW, Behrens TEJ, Smith SM, Johansen-Berg H (2012) Tools of the trade: psychophysiological interactions and functional connectivity. Soc Cogn Affect Neurosci 7:604-609.

Okuda J, Fujii T, Ohtake H, Tsukiura T, Tanji K, Suzuki K, Kawashima R, Fukuda H, Itoh M, Yamadori A (2003) Thinking of the future and past: The roles of the frontal pole and the medial temporal lobes. Neuroimage 19:1369-1380.

Ongür D, Price JL (2000) The organization of networks within the orbital and medial prefrontal cortex of rats, monkeys and humans. Cereb Cortex 10:206-219.

Poppenk J, Evensmoen HR, Moscovitch M, Nadel L (2013) Long-axis specialization of the human hippocampus. Trends Cogn Sci 17:230-240.

Power JD, Barnes KA, Snyder AZ, Schlaggar BL, Petersen SE (2012) Spurious but 
systematic correlations in functional connectivity MRI networks arise from subject motion. Neuroimage 59:2142-2154.

Preston AR, Eichenbaum H (2013) Interplay of hippocampus and prefrontal cortex in memory. Curr Biol 23:R764-R773.

Preston AR, Shrager Y, Dudukovic N, Gabrieli JDE (2004) Hippocampal contribution to the novel use of relational information in declarative memory. Hippocampus $14: 148-152$.

Price JL, Drevets WC (2009) Neurocircuitry of mood disorders. Neuropsychopharmacology 35:192-216.

Richter FR, Chanales AJH, Kuhl BA (2015) Predicting the integration of overlapping memories by decoding mnemonic processing states during learning. Neuroimage.

Roy M, Shohamy D, Wager TD (2012) Ventromedial prefrontal-subcortical systems and the generation of affective meaning. Trends Cogn Sci 16:147-156.

Schacter DL, Addis DR, Hassabis D, Martin VC, Spreng RN, Szpunar KK (2012) The future of memory: remembering, imagining, and the brain. Neuron 76:677-694.

Schedlbauer AM, Copara MS, Watrous AJ, Ekstrom AD (2014) Multiple interacting brain areas underlie successful spatiotemporal memory retrieval in humans. Sci Rep 4:6431.

Schlichting ML, Mumford JA, Preston AR (2015) Learning-related representational changes reveal dissociable integration and separation signatures in the hippocampus and prefrontal cortex. Nat Commun 6:8151.

Schlichting ML, Preston AR (2014) Memory reactivation during rest supports upcoming learning of related content. Proc Natl Acad Sci U S A 111:15845-15850.

Schlichting ML, Preston AR (2015) Memory integration: neural mechanisms and implications for behavior. Curr Opin Behav Sci 1:1-8.

Schnider A (2003) Spontaneous confabulation and the adaptation of thought to ongoing reality. Nat Rev Neurosci 4:662-671.

Schonberg T, Bakkour A, Hover AM, Mumford JA, Nagar L, Perez J, Poldrack RA (2014) Changing value through cued approach: an automatic mechanism of behavior change. Nat Neurosci 17:625-630.

Smith SM, Jenkinson M, Johansen-Berg H, Rueckert D, Nichols TE, Mackay CE, Watkins KE, Ciccarelli O, Cader MZ, Matthews PM, Behrens TEJ (2006) Tractbased spatial statistics: voxelwise analysis of multi-subject diffusion data. 
Neuroimage 31:1487-1505.

St. Jacques PL, Olm C, Schacter DL (2013) Neural mechanisms of reactivation-induced updating that enhance and distort memory. Proc Natl Acad Sci U S A 110:1967119678.

Staresina BP, Alink A, Kriegeskorte N, Henson RN (2013) Awake reactivation predicts memory in humans. Proc Natl Acad Sci U S A 110:21159-21164.

Sweegers CCG, Takashima A, Fernández G, Talamini LM (2013) Neural mechanisms supporting the extraction of general knowledge across episodic memories. Neuroimage 87:138-146.

Tambini A, Ketz N, Davachi L (2010) Enhanced brain correlations during rest are related to memory for recent experiences. Neuron 65:280-290.

Tse D, Langston RF, Kakeyama M, Bethus I, Spooner PA, Wood ER, Witter MP, Morris RGM (2007) Schemas and Memory Consolidation. Science 316:76-82.

Tse D, Takeuchi T, Kakeyama M, Kajii Y, Okuno H, Tohyama C, Bito H, Morris RGM (2011) Schema-Dependent Gene Activation and Memory Encoding in Neocortex. Science 333:891-895.

van Kesteren MTR, Fernández G, Norris DG, Hermans EJ (2010a) Persistent schemadependent hippocampal-neocortical connectivity during memory encoding and postencoding rest in humans. Proc Natl Acad Sci U S A 107:7550-7555.

van Kesteren MTR, Rijpkema M, Ruiter DJ, Fernandez G (2010b) Retrieval of associative information congruent with prior knowledge is related to increased medial prefrontal activity and connectivity. J Neurosci 30:15888-15894.

van Kesteren MTR, Rijpkema M, Ruiter DJ, Morris RGM, Fernández G (2014) Building on prior knowledge: Schema-dependent encoding processes relate to academic performance. J Cogn Neurosci 26:2250-2261.

van Kesteren MTR, Ruiter DJ, Fernández G, Henson RN (2012) How schema and novelty augment memory formation. Trends Neurosci 35:211-219.

Warren DE, Jones SH, Duff MC, Tranel D (2014) False recall is reduced by damage to the ventromedial prefrontal cortex: Implications for understanding the neural correlates of schematic memory. J Neurosci 34:7677-7682.

Wierzynski CM, Lubenov E V., Gu M, Siapas AG (2009) State-Dependent Spike-Timing Relationships between Hippocampal and Prefrontal Circuits during Sleep. Neuron 61:587-596. 
Worsley KJ, Liao CH, Aston J, Petre V, Duncan GH, Morales F, Evans AC (2002) A general statistical analysis for fMRI data. Neuroimage 15:1-15.

Zeithamova D, Dominick AL, Preston AR (2012a) Hippocampal and ventral medial prefrontal activation during retrieval-mediated learning supports novel inference. Neuron 75:168-179.

Zeithamova D, Preston AR (2010) Flexible memories: Differential roles for medial temporal lobe and prefrontal cortex in cross-episode binding. J Neurosci 30:1467614684.

Zeithamova D, Schlichting ML, Preston AR (2012b) The hippocampus and inferential reasoning: Building memories to navigate future decisions. Front Hum Neurosci 6:70. 


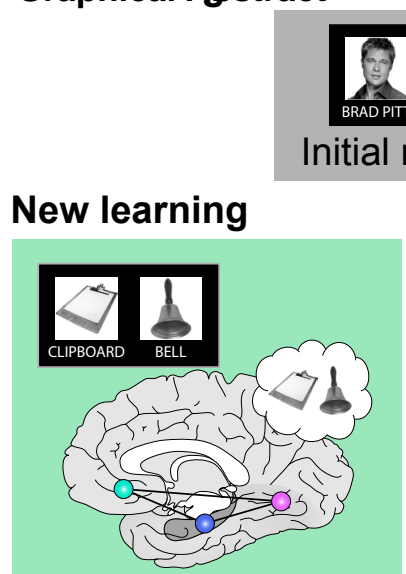

No updating

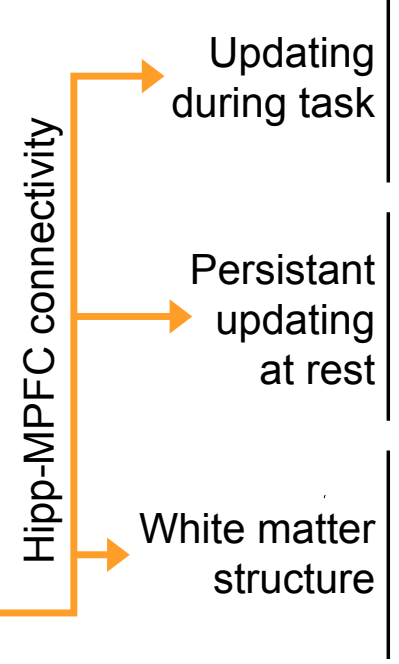

